\title{
Seismic Response Reduction of Structures Equipped with a Voided Biaxial Slab-Based Tuned Rolling Mass Damper
}

\author{
Shujin Li, ${ }^{1}$ Liming Fu, ${ }^{1,2}$ and Fan Kong ${ }^{1}$ \\ ${ }^{1}$ School of Civil Engineering and Architecture, Wuhan University of Technology, 122 Luoshi Road, Wuhan 430070, China \\ ${ }^{2}$ Hubei Synthetic Space Building Technology Co. Ltd., Wuhan 430070, China \\ Correspondence should be addressed to Fan Kong; kongfan@whut.edu.cn
}

Received 25 February 2015; Accepted 14 May 2015

Academic Editor: Kumar V. Singh

Copyright (c) 2015 Shujin Li et al. This is an open access article distributed under the Creative Commons Attribution License, which permits unrestricted use, distribution, and reproduction in any medium, provided the original work is properly cited.

\begin{abstract}
This paper proposes a novel tuned mass damper (TMD) embedded in hollow slabs of civil structures. The hollow slabs in this context, also referred to as "voided biaxial reinforced concrete slabs," feature a large interior space of prefabricated voided modules that are necessary in the construction of this special structural system. In this regard, a tuned rolling mass damper system (“TRoMaDaS") is newly proposed, in combination with hollow slabs, to act as an ensemble passive damping device mitigating structural responses. The main advantage of this TMD configuration lies in its capacity to maintain architectural integrity. To further investigate the potential application of the proposed TRoMaDaS in seismic response mitigation, theoretical and numerical studies, including deterministic and stochastic analyses, were performed. They were achieved by deterministic dynamic modeling using Lagrange's equation and the statistical linearization method. Finally, the promising control efficacy obtained from the deterministic/stochastic analysis confirmed the potential application of this newly proposed control device.
\end{abstract}

\section{Introduction}

Civil structures exposed to ambient dynamic excitation often exhibit excessive responses that need to be mitigated, from considerations of safety and/or serviceability issues of the structures. Traditionally, relying on the enhancement of material strength and cross-sectional area, structure/element responses can be reduced to some level. However, this treatment of suppressing structure/element responses is not economically feasible, especially for structures located in the disastrous earthquake- or gust-prone areas. An alternative and smarter measure to suppress structural response is socalled structural control.

Begun by Yao [1], structural control techniques are intended to mitigate structure responses to random ambient excitation. They have been developed for decades and some of them have matured with large advances in both theoretical and practical aspects. The central concept of structural control theory, in fact, relies on the changing of structural parameters or external excitations appropriately, passively, actively, semiactively, or with combinations of them, leading to mitigated structure responses. To date, many research papers and several important textbooks have been published in this area, such as $[2,3]$. Among these structural control methods, the most widely used and also most mature is passive control, whereas active control and semiactive control suffer from various practical or theoretical challenges that need further investigation.

Tuned mass dampers (TMDs), attached to the primary structure, consist of a block of mass, a stiffness-restoring element, and an energy dissipation element. These are now one of the most widely used passive control devices, especially against wind-induced vibration in high-rise buildings, because of their advantages in terms of operation, maintenance, and fabrication (see [4] for details).

However, the traditional configuration of the TMDs requires dedicated large space to accommodate the huge scale of the mass and the corresponding functional components providing damping and stiffness. As an example, the Chifley Tower in Sydney, a 52-storey $209-\mathrm{m}$ tall steel structure, is fitted with a single pendulum-type TMD suspended by steel cables at the 44th floor [4]. The $400 \mathrm{t}$ block of steel with dimensions of $4 \times 4 \times 4 \mathrm{~m}$ and the stroke (about $\pm 910 \mathrm{~mm}$ ) of the mass indicates the size of the dedicated space needed. 
Moreover, the suspension cables need to stretch across two floors (from the 44th to the 46th floors) to tune the natural frequency of the damper to the frequency of the tower. Other examples include the platform-type TMD system, consisting of a concrete mass of $373 \mathrm{t}$ (about $150 \mathrm{~m}^{3}$ ) with a stroke of $\pm 1.14 \mathrm{~m}$, employed at the 63rd floor of the $278 \mathrm{~m}$ tall Citicorp Center in New York City [4]. Even for the more compact configuration of the inverted pendulum-type TMD installed at the $100 \mathrm{~m}$ level of the $134 \mathrm{~m}$ high Sky Tower in Nagoya [5], Japan, the dimensions of the mass block are $2.5 \mathrm{~m}$ square $\times$ $3 \mathrm{~m}$, with a stroke of $150 \mathrm{~mm}$. Furthermore, the installation of a TMD as an additional component leads to, in some sense, an inconsistency in architectural styles.

Recently, a structure system with a voided biaxial slab has been used widely in practical engineering, especially in Europe [6, 7] and China [8]. Originating from onespanning hollow-core slabs, recent developments on voided slab technology have been aimed at reducing the dead weight and thus enhancing the span of the structure floors. Specifically, this is achieved by laying less heavy material, such as polystyrene or polypropylene made hollow modules between the reinforcement of beams/ribbed beams, and thus displacing concrete with less structural benefit. A typical technology used in Europe is the so-called "BubbleDeck" biaxial voided slab $[9,10]$, in which spherical hollow modules composed of recycled industrial plastic are used, allowing the hollow slab to act as a normal monolithic two-way spanning concrete slab. For another form of hollow module, see the "Uboot" [7] technology, as an example. One of the main features of these hollow modules is the large voided interior space.

In this context, we propose a novel form of distributed TMD that we have named the tuned rolling mass damper system (“TRoMaDaS") for this specific structure, taking advantage of the large space in the voided module that has not before been considered for structural control. Specifically, the rolling ball TMD, first suggested by Pirner [11] and Náprstek and colleagues [12] is, for the first time, proposed to be distributed in the hollow floors of the structures. This arrangement of the TMDs is expected to not only suppress the structure response but also maintain the consistency of the architectural style in the sense of architectural esthetics.

To validate the control efficacy of the proposed passive control system, a physical and mathematical model of the controlled chain-like MDOF structure was developed. In this regard, Lagrange's equation was used to derive the coupled equations of motion of the controlled system, in which the stroke of the rolling mass is considered a small quantity and nonlinearity is exhibited in the damping force. The derivation of the dynamic equation from the physical model is quite general, in which any TRoMaDaS with arbitrary parameters can be considered. The Runge-Kutta method of the fourth order was used to solve the dynamic equations numerically for cases with different TRoMaDaS parameters or distributions. We conclude that, for all cases, with the equipment of the proposed TRoMaDaS, the input energy of an ambient excitation is absorbed to a large degree. Furthermore, it has been found that because of the "stickslip" behavior of friction-type TMDs [13], the peak value of response cannot be efficiently reduced, whereas the postpeak response can, in the context of earthquake excitations. In this study, we also show that different vertical distributions and parameter configurations of the TRoMaDaS lead to different control efficiencies, necessitating a more complete investigation of the optimum design of TRoMaDaS in future studies. Finally, to investigate the control efficiency from a probabilistic perspective, a statistical linearization method, along with a pertinent Monte Carlo simulation, was used to calculate the standard deviation of the response displacement of a structure with and without the proposed TRoMaDaS. The stochastic analysis of the structure revealed that not only was the displacement standard deviation reduced to a large degree but also the stochastic response of the structure readily became stationary because of the TRoMaDaS.

\section{Voided Biaxial Slab-Based Tuned Rolling Mass Damper}

2.1. Structural System with Voided Biaxial Slabs. The voided biaxial reinforced concrete slab, constructed using prefabricated voided modules and cast in situ reinforced concrete, has gained wide attention since its emergence as a new structural system [6-10]. These ecofriendly voided biaxial slabs have many advantages over conventional solid concrete slabs, such as lower total cost, reduced material use, enhanced structural efficiency, and decreased construction time, that have contributed to their wide application in civil construction.

Taking one kind of voided biaxial slab used widely in China as an example, the construction procedures for this structural system are as follows. First, the prefabricated hollow box-like modules are located between the reinforcement grids of the main beams and the ribbed beams. Next, boxlike voided modules are used as the side formwork when site-casting the concrete beams. In this way, only the bottom formworks of the concrete slab are needed, thus saving considerable construction costs. To guarantee the integrity between the prefabricated hollow modules and the circumjacent reinforced concrete, nonstructural measures, such as extending the reinforcement bar into the main beams/ribbed beams, may be implemented. The vertical dimension of a prefabricated hollow module, according to the spans of main beams, ranges from 200 to $900 \mathrm{~mm}$. Figure 1 shows the construction procedures of a kind of voided biaxial slab in detail.

\subsection{Motivation of Voided Biaxial Slab-Based Tuned Rolling} Mass Damper. We noted the unique large space in the hollow modules and thus proposed to locate certain structural or architectural elements within these modules. Specifically, these additional elements can be designed as a part of heating, ventilation and air conditioning, water supply and sewerage, and structural control systems, without altering the architectural appearance excessively. Among these architectural and structural requirements, structural safety and serviceability are the most important issues. In this regard, we proposed to install passive control devices in the hollow modules to mitigate the dynamic responses of the structure.

Among the passive control devices, the passive vibration absorber or the tuned mass damper (TMD) has been 


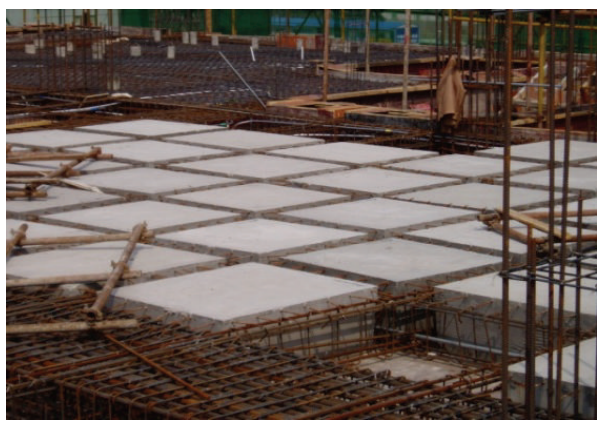

(a)

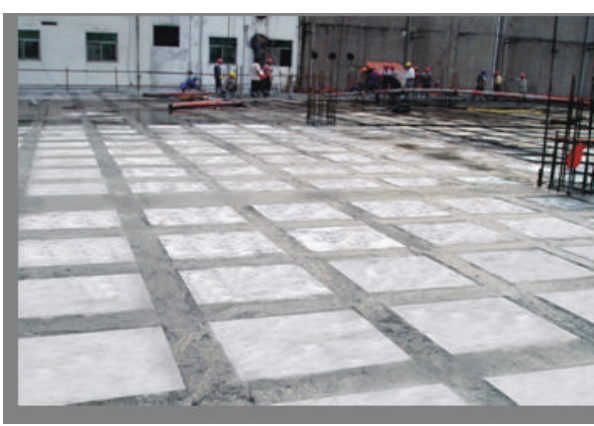

(b)

Figure 1: Construction procedures for a hollow-ribbed floor. (a) Locate prefabricated hollow box-like modules between the reinforcement grids. (b) Cast reinforced concrete beams between the box-like hollow modules.

researched widely and used extensively in newly constructed buildings and in the retrofitting of existing structures, because of its simplicity, relatively low construction and maintenance costs, and safety. In this regard, TMDs of the pendulum type [4] are the most widely used, especially for slender towers and high-rise buildings. However, this configuration requires dedicated large vertical and horizontal dimensions to accommodate the extra weight, as mentioned in Section 1, especially for high-rise buildings with a low natural fundamental frequency. Moreover, safety measures, such as mechanical stops or other braking systems, are also required to limit the excessive travel of the mass block.

An alternative TMD configuration is the type mounted on a large complex mechanical platform $[14,15]$, which provides damping and stiffness using hydrostatic bearings, hydraulic cylinders, pneumatic springs, or coil springs. It can be argued that, compared with TMDs of the pendulum type, the platform-type has increased complexity and thus costs more in terms of daily operations and maintenance. Thus, we concluded that neither of these TMD configurations was suitable for a vibration mitigation device to be installed in the modules of the voided biaxial slab.

Another more compact and also simple configuration is the one with an object rolling on a tridimensional surface. The damping mechanism and restoring force of this configuration are provided by the rolling friction and the resulting force of the gravity of the rolling mass, respectively. Specifically, the so-called tuned rolling mass damper (TRMD) or the ball vibration absorber (BVA) consists of a ball rolling along an arch path located in hollow modules to absorb the structural kinetic and potential energy as the result of the ambient excitations. In fact, the TRMD has been applied in several other engineering structures, such as long-span bridges [16], TV towers [11], and wind turbines [17, 18]. However, to our knowledge, very few applications of TRMDs or BVAs in building structures to suppress seismic vibrations have been reported, except those proposed recently by Fisher and Pirner [19] and Matta et al. [20]. Moreover, compared with the "traditional" configuration of TMDs in high-rise buildings, where the TMDs are always installed as an additional energyabsorbing element, the most remarkable advantage of the installation of TRMDs in hollow modules embedded in slabs

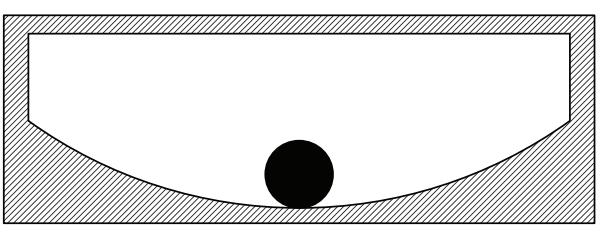

Figure 2: Voided biaxial slab-based tuned rolling mass damper.

lies in its architectural integrity. By locating the passive control device in an existing space (shown in construction site artwork; Figure 1), installation of the voided biaxial slabbased tuned rolling mass damper system ("VBS-TRoMaDaS" or "TRoMaDaS") does not alter the architectural interior appearance excessively. A diagram of a TRoMaDaS with a single rectangular hollow module is shown in Figure 2.

2.3. Characteristics of TRoMaDaS. By appropriately selecting parameters, including the radius of the arc path and the oscillator (the natural frequency of the TRoMaDaS depends on these parameters as shown in Section 3), the friction coefficient between the arc path and the oscillator (the damping capacity of the TRoMaDaS depends on this), and the material/density of the oscillator, an optimum controlled structure can be obtained. From the review above, it can be observed that, compared with pendulum-type and platform-type TMDs, the energy dissipation mechanism of the TRoMaDaS is more compact and simpler. These promising features, naturally, lead to a more durable passive control system and reduce considerably the cost of fabrication, maintenance, and operation. Moreover, the proposed TRoMaDaS is expected to suppress the coupled lateral and torsional motions of structures, due to accidental or intended eccentricities between their mass and stiffness centers. In this regard, Singh et al. [21] investigated the efficiency of four TMDs of the dashpot-spring type, placed along two orthogonal directions in pairs, to mitigate the torsionally coupled responses of irregular multistorey buildings. Jangid and Datta [22] conducted a parametric study of the effectiveness of multi-TMDs along the width of an eccentric structure in reducing torsionally coupled responses. However, both 


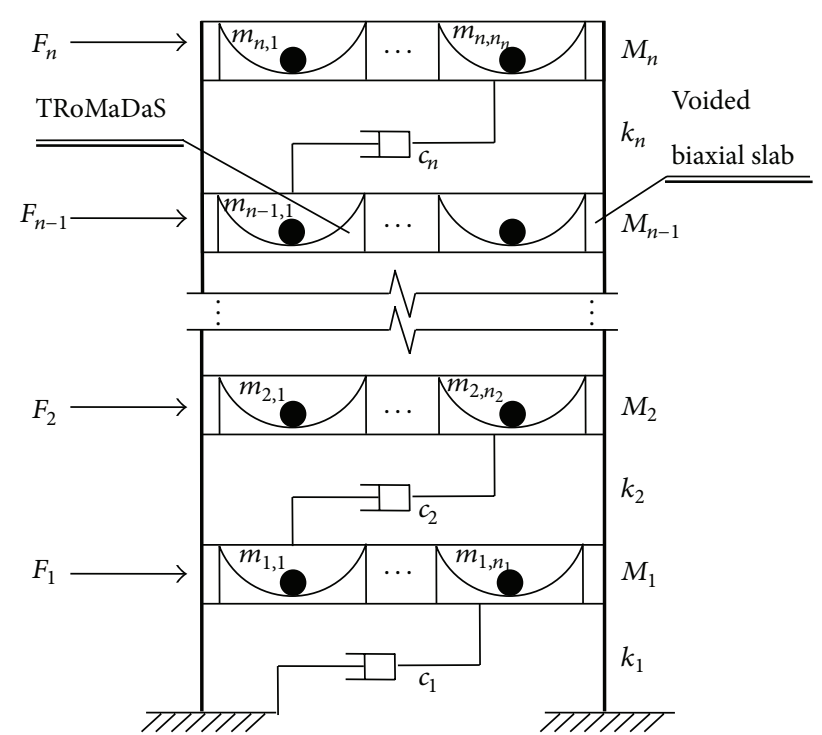

FIGURE 3: Lumped-mass model of a shear-type structure equipped with a TRoMaDaS.

investigations focused on a theoretical analysis of mitigating torsionally coupled responses using ideal physical models, whereas the practical feasible multi-TMDs configuration has not been considered.

In this aspect, the proposed voided biaxial slab-based TRoMaDaS can be used as a platform to realize optimally controlled structures with various damper distributions/arrangement and parameters. Specifically, because there is no restriction on the direction of the rolling motion or the location/distribution of the oscillator, the proposed TRoMaDaS can, in fact, be fabricated as a type of spatial multi-TMDs system that can be used to control structure motion in every direction and even torsional motion caused by eccentric structures or bidirectional base excitations. Moreover, to fully use the spatial movement of the oscillator, a three-dimensional TRoMaDaS with a nonaxially symmetrical three-dimensional path surface can be used to control motions of spatial structures with different natural mode frequencies in two orthogonal directions simultaneously. This concept was initially proposed by Matta et al. [20] in the context of rolling-pendulum TMDs and can be used to extend the possible application of the TRoMaDaS in structures with different frequencies in two orthogonal directions.

\section{Equation of Motion of a Controlled Structure with TRoMaDaS}

3.1. Simplified Model of a TRoMaDaS-Controlled MDOF Structure. Consider a mass-lumped model of a shear-type structure equipped with a TRoMaDaS in each hollow floor (Figure 3), where $\left(M_{1}, M_{2}, \ldots, M_{n}\right),\left(k_{1}, k_{2}, \ldots, k_{n}\right)$, and $\left(c_{1}, c_{2}, \ldots, c_{n}\right)$ are the mass, stiffness, and the damping coefficient of the main structure, respectively, and $F_{j}, j=$ $1,2, \ldots, n$, are the external ambient excitations, including the equivalent force of base excitation or wind loading. For

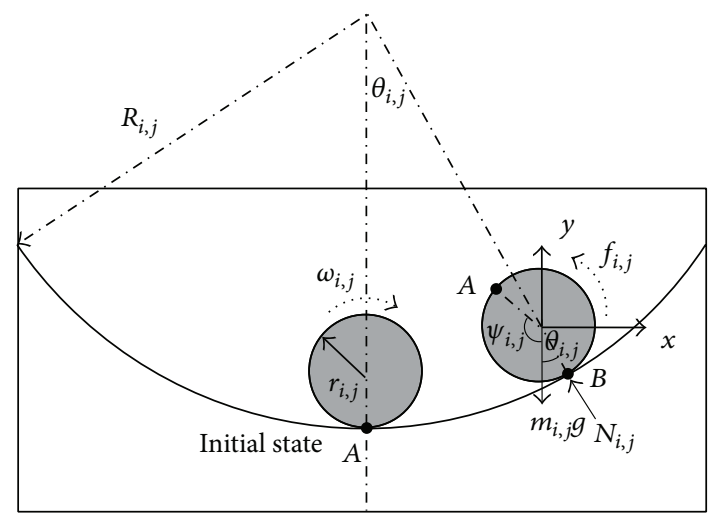

FIgURE 4: Free-body diagram of a TRoMaDaS.

simplicity, the TRoMaDaS in each floor contains several oscillators with different masses $\left(m_{i, 1}, m_{i, 2}, \ldots, m_{i, n_{i}}\right)$ rolling along the axial-symmetrical sphere surfaces, where $m_{i, j}$ represents the $j$ th oscillator located on the $i$ th floor. From Figure 3, it can be seen that the total degrees of freedom are $n+\left(n_{1}+n_{2}+\cdots+n_{n}\right)$, with $n$ degrees of freedom of translational motion of the lumped mass and $\left(n_{1}+n_{2}+\cdots+n_{n}\right)$ degrees of freedom of the oscillators' rotation with respect to the centers of the arch paths, respectively. A free-body diagram of the TRoMaDaS is shown in Figure 4 , where $R_{i, j}$, $r_{i, j}, i=1,2, \ldots, n_{i}, j=1,2, \ldots, n_{i}$, are the radii of the $(i, j)$ th arc path and oscillator, respectively, $\theta_{i, j}, \psi_{i, j}$ are the rotation angles of the oscillator with respect to the center of the path and of the oscillator ball itself, and $\omega_{i, j}=\dot{\psi}_{i, j}$ denotes the angular velocity of the oscillator.

3.2. Governing Equation of the TRoMaDaS-Controlled Structure. Thus, the kinetic and potential energy of the controlled structure can be written as

$$
\begin{array}{r}
T=\sum_{i=1}^{n} \sum_{j=1}^{n_{i}}\left\{\frac{1}{2} m_{i, j}\left[\dot{x}_{i}+\rho_{i, j} \dot{\theta}_{i, j} \cos \theta_{i, j}\right]^{2}\right. \\
\left.+\frac{1}{2} m_{i, j}\left[\rho_{i, j} \dot{\theta}_{i, j} \sin \theta_{i, j}\right]^{2}+\frac{1}{2} J_{i, j} \omega_{i, j}^{2}+\frac{1}{2} M_{i} \dot{x}_{i}^{2}\right\}, \\
i=1,2, \ldots, n ; j=1,2, \ldots, n_{i}, \\
V=\sum_{i=1}^{n} \frac{1}{2} k_{i}\left(x_{i}-x_{i-1}\right)^{2}+\sum_{i=1}^{n} \sum_{j=1}^{n_{i}} m_{i, j} g \rho_{i, j}\left(1-\cos \theta_{i, j}\right), \\
i=1,2, \ldots, n ; j=1,2, \ldots, n_{i},
\end{array}
$$

respectively, where $x_{i}, \dot{x}_{i}$ are the translational displacement and velocity of the lumped mass, $\rho_{i, j}=R_{i, j}-r_{i, j}$ is the radius difference between the arc path and the oscillator, $\dot{\theta}_{i, j}$ is the angular velocity of the oscillator with respect to the center of the arc path, and $J_{i, j}=2 m_{i, j} r_{i, j}^{2} / 5$ is the rotational inertia of 
the $j$ th oscillator on the $i$ th floor. Furthermore, (1)-(2) can be simplified to

$$
\begin{gathered}
T=\sum_{i=1}^{n} \sum_{j=1}^{n_{i}} \frac{1}{2} m_{i, j}\left(\dot{x}_{i}^{2}+2 \dot{x}_{i} \rho_{i, j} \dot{\theta}_{i, j}+\rho_{i, j}^{2} \dot{\theta}_{i, j}^{2}\right) \\
+\frac{1}{5} m_{i, j} \rho_{i, j}^{2} \dot{\theta}_{i, j}^{2}+\frac{1}{2} M_{i} \dot{x}_{i}^{2}, \\
i=1,2, \ldots, n ; j=1,2, \ldots, n_{i}, \\
V=\sum_{i=1}^{n} \sum_{j=1}^{n_{i}}\left[\frac{1}{2} k_{j}\left(x_{j}-x_{j-1}\right)^{2}+\frac{1}{2} m_{i, j} g \rho_{i, j} \theta_{i, j}^{2}\right], \\
i=1,2, \ldots, n ; j=1,2, \ldots, n_{i},
\end{gathered}
$$

when the angular motion of the oscillator $\left(\theta_{j}\right)$ is considered to be small. Note in (3) that the compatibility condition of displacement $R_{i, j} \theta_{i, j}=\left(\theta_{i, j}+\psi_{i, j}\right) r_{i, j}$ is used. It may be argued that the small-quantity assumption for the oscillator response and (3) tend to be reasonable in cases of lower-level external excitations. However, it should be noted that not only may the structure undergo nonlinear behavior but also the oscillator response may surpass a certain small-quantity level in the case of intense excitations. Although the smallquantity assumption is important and often used in the simplification of governing equations of pendulum- or rollingpendulum-type TMDs, further examination of the applicability of this assumption during intense external excitation is necessary.

The nonconservative force $Q_{j}^{\text {nc }}$, including the external force, the damping force of the structure, and the rolling friction force between the sphere surface and the oscillator, can be derived by the virtual work principle; that is,

$$
\begin{aligned}
\delta W & =\sum_{i=1}^{n}\left\{F_{i} \delta x_{i}\right. \\
+ & {\left.\left[-\left(\dot{x}_{i}-\dot{x}_{i-1}\right) c_{i}+\left(\dot{x}_{i+1}-\dot{x}_{i}\right) c_{i+1}\right] \delta x_{i}\right\} } \\
& -\sum_{i=1}^{n} \sum_{j=1}^{n_{i}} f_{i, j} \frac{\delta s_{i, j}}{r_{i, j}}, \quad i=1,2, \ldots, n ; j=1,2, \ldots, n_{i},
\end{aligned}
$$

where $\delta W$ denotes the total virtual work of the nonconservative force, $s_{i, j}=R_{i, j} \theta_{i, j}=r_{i, j}\left(\psi_{i, j}+\theta_{i, j}\right)$ is the arc length of the oscillator movements between the initial state and state being considered, and $f_{i, j}$ is the moment of the rolling friction force with respect to center of the oscillator and can be determined by

$$
\begin{aligned}
f_{i, j} & =-\frac{\dot{\theta}_{i, j}}{\left|\dot{\theta}_{i, j}\right|} \mu_{i, j} N_{i, j} \\
& =-\frac{\dot{\theta}_{i, j}}{\left|\dot{\theta}_{i, j}\right|} \mu_{i, j} m_{i, j}\left(g \cos \theta_{i, j}+\rho_{i, j} \dot{\theta}_{i, j}^{2}\right) .
\end{aligned}
$$

In (5), $N_{i, j}$ is the $j$ th reaction force perpendicular to the tangent of the contact point on the $i$ th floor and $\mu_{i, j}$ is the coefficient of the rolling friction (in meters) between the $j$ th oscillator and the sphere surface on the $i$ th floor. Combining (4)-(5), the nonconservative force can be written as

$$
\begin{aligned}
& Q_{i}^{\mathrm{nc}, \mathrm{s}}=F_{i}+\left[-\left(\dot{x}_{i}-\dot{x}_{i-1}\right) c_{i}+\left(\dot{x}_{i+1}-\dot{x}_{i}\right) c_{i+1}\right], \\
& i=1,2, \ldots, n, \\
& Q_{i, j}^{\mathrm{nc}, \mathrm{o}}=-\frac{\dot{\theta}_{i, j} R_{i, j}}{\left|\dot{\theta}_{i, j}\right| r_{i, j}} \mu_{i, j} m_{i, j} g, \\
& \quad i=1,2, \ldots, n ; j=1,2, \ldots, n_{i} .
\end{aligned}
$$

In fact, (6) is the expression for the viscous damping force and the external excitation of main structures, whereas (7) denotes the rolling friction force between the oscillator and the sphere surface.

The governing equation of the controlled structure can be derived by invoking the Lagrange equation:

$$
\begin{aligned}
\frac{\mathrm{d}}{\mathrm{d} t}\left(\frac{\partial T}{\partial \dot{q}_{i}}\right)-\frac{\partial T}{\partial q_{i}}+\frac{\partial V}{\partial q_{i}} & =Q_{i}^{\mathrm{nc}}, \\
& \quad i=1,2, \ldots, 2 n+\left(n_{1}+n_{2}+\cdots+n_{n}\right),
\end{aligned}
$$

where $q_{i}, \dot{q}_{i}$ are the generalized displacement and velocity of the $i$ th coordinate. Combining (3) and (8) and considering the angular displacement and velocity $\left(\theta_{j}, \dot{\theta}_{j}\right)$ to be small quantities, one can obtain

$$
\begin{gathered}
\left(M_{i}+\sum_{j=1}^{n_{i}} m_{i, j}\right) \ddot{x}_{i}+\sum_{j=1}^{n_{i}} m_{i, j} \rho_{i, j} \ddot{\theta}_{i, j}+\left(x_{i}-x_{i-1}\right) k_{i} \\
-\left(x_{i+1}-x_{i}\right) k_{i+1}+\left(\dot{x}_{i}-\dot{x}_{i-1}\right) c_{i}-\left(\dot{x}_{i+1}-\dot{x}_{i}\right) c_{i+1} \\
=F_{i}, \\
\ddot{\theta}_{i, j}+\frac{5 \mu_{i, j} g R_{i, j}}{7 \rho_{i, j}^{2} r_{i, j}} \operatorname{sgn}\left(\dot{\theta}_{i, j}\right)+\frac{5 g \theta_{i, j}}{7 \rho_{i, j}}=-\frac{5}{7 \rho_{i, j}} \ddot{x}_{i, j}, \\
i=1,2, \ldots, n ; j=1,2, \ldots, n_{i},
\end{gathered}
$$

where $\operatorname{sgn}(\cdot)$ denotes the sign function. Equations (9a) and (9b) are a set of coupled nonlinear ordinary differential equations, even with the small-quantity assumption of the rolling motion. On the left side of (9a), the terms $\ddot{x}_{i} \sum_{j=1}^{n_{i}} m_{i, j}+$ $\sum_{j=1}^{n_{i}} m_{i, j} \rho_{i, j} \ddot{\theta}_{i, j}$ can be regarded as the control force produced by the translational and rotational motion of the oscillator, counteracting part of the external force, $F_{i}$. Moreover, the right side of (9b) shows that the motion of the oscillators is caused by the supported base acceleration, which is the acceleration of the main structure. The left side of (9b) shows the undamped natural frequency of the $j$ th oscillator on the $i$ th floor depends only on the radius difference between the arch path and the oscillator and can be written as

$$
\omega_{i, j}=\sqrt{\frac{5 g}{7 \rho_{i, j}}} .
$$


This expression is consistent with that derived by Zhang and colleagues [17] and Chen and Georgakis [18]. Equations (9a) and $(9 \mathrm{~b})$ can be rewritten in a compact form:

$$
\mathbf{M} \ddot{\mathbf{z}}+\mathbf{C} \dot{\mathbf{z}}+\mathbf{K z}+\mathbf{f}(\mathbf{z}, \dot{\mathbf{z}})=\mathbf{w}(t),
$$

where

$$
\begin{aligned}
& \mathbf{M}=\left[\begin{array}{ll}
\mathbf{M}_{1} & \mathbf{M}_{2} \\
\mathbf{M}_{3} & \mathbf{M}_{4}
\end{array}\right], \\
& \mathbf{C}=\left[\begin{array}{ll}
\mathbf{C}_{1} & \mathbf{0} \\
\mathbf{0} & \mathbf{0}
\end{array}\right], \\
& \mathbf{K}=\left[\begin{array}{cc}
\mathbf{K}_{1} & 0 \\
0 & \mathbf{K}_{2}
\end{array}\right]
\end{aligned}
$$

are the mass, damping, and stiffness coefficients; the augmented displacement is $\mathbf{z}=\left[\mathbf{x}^{T}, \boldsymbol{\theta}_{1}^{T}, \boldsymbol{\theta}_{2}^{T}, \ldots, \boldsymbol{\theta}_{n}^{T}\right]^{T}$ with $\mathbf{x}=$ $\left(x_{1}, x_{2}, \ldots, x_{n}\right)^{T}, \boldsymbol{\theta}_{1}=\left(\theta_{1,1}, \theta_{1,2}, \ldots, \theta_{1, n_{1}}\right)^{T}, \boldsymbol{\theta}_{2}=\left(\theta_{2,1}, \theta_{2,2}\right.$, $\left.\ldots, \theta_{2, n_{2}}\right)^{T}$, and $\boldsymbol{\theta}_{n}=\left(\theta_{n, 1}, \theta_{n, 2}, \ldots, \theta_{n, n_{n}}\right)^{T}$; the external excitation on the structure at the right side of (11) is $\mathbf{w}=$ $[\mathbf{w}_{s}^{T}, \underbrace{0,0, \ldots, 0}_{n_{1}+n_{2}+\cdots+n_{n}}]^{T}$ with $\mathbf{w}_{s}=\left(F_{1}, F_{2}, \ldots, F_{n}\right)^{T}$, and the nonlinear damping force can be written as

$$
\begin{aligned}
& f(\mathbf{z}, \dot{\mathbf{z}})=[\underbrace{0,0, \ldots, 0}_{n} ; \frac{5 \mu_{1,1} g R_{1,1}}{7 \rho_{1,1}^{2} r_{1,1}} \\
& \cdot \operatorname{sgn}\left(\dot{\theta}_{1,1}\right), \frac{5 \mu_{1,2} g R_{1,2}}{7 \rho_{1,2}^{2} r_{1,2}} \operatorname{sgn}\left(\dot{\theta}_{1,2}\right), \ldots, \frac{5 \mu_{1, n_{1}} g R_{1, n_{1}}}{7 \rho_{1, n_{1}}^{2} r_{1, n_{1}}} \\
& \cdot \operatorname{sgn}\left(\dot{\theta}_{1, n_{1}}\right) ; \ldots ; \frac{5 \mu_{n, 1} g R_{n, 1}}{7 \rho_{n, 1}^{2} r_{n, 1}} \operatorname{sgn}\left(\dot{\theta}_{n, 1}\right), \frac{5 \mu_{n, 2} g R_{n, 2}}{7 \rho_{n, 2}^{2} r_{n, 2}} \\
& \left.\cdot \operatorname{sgn}\left(\dot{\theta}_{n, 2}\right), \ldots, \frac{5 \mu_{n, n_{n}} g R_{n, n_{n}}}{7 \rho_{n, n_{n}}^{2} r_{n, n_{n}}} \operatorname{sgn}\left(\dot{\theta}_{n, n_{n}}\right)\right] .
\end{aligned}
$$

Furthermore, submatrices in (12) are

$$
\begin{aligned}
\mathbf{M}_{1} & =\operatorname{diag}\left(M_{1}+\sum_{j=1}^{n_{1}} m_{1, j}, M_{2}+\sum_{j=1}^{n_{2}} m_{2, j}, \ldots, M_{n}\right. \\
& \left.+\sum_{j=1}^{n_{n}} m_{n, j}\right) \\
\mathbf{M}_{2} & =\operatorname{diag}\left(\mathbf{M}_{2}^{1}, \mathbf{M}_{2}^{2}, \ldots, \mathbf{M}_{2}^{n}\right), \\
\mathbf{M}_{2}^{i} & =\left(m_{i, 1} \rho_{i, 1}, m_{i, 2} \rho_{i, 2}, \ldots, m_{i, n_{i}} \rho_{i, n_{i}}\right)_{1 \times n_{i}}, \\
\mathbf{M}_{3} & =\operatorname{diag}\left(\mathbf{M}_{3}^{1}, \mathbf{M}_{3}^{2}, \ldots, \mathbf{M}_{3}^{n}\right), \\
\mathbf{M}_{3}^{i} & =\frac{5}{7}\left(\frac{1}{\rho_{i, 1}}, \frac{1}{\rho_{i, 2}}, \ldots, \frac{1}{\rho_{i, n_{i}}}\right)_{n_{i} \times 1}^{T},
\end{aligned}
$$

$$
\begin{aligned}
& \mathbf{M}_{4}=\mathbf{I} \text {, } \\
& \mathbf{K}_{2}=\operatorname{diag}\left(\mathbf{K}_{2}^{1}, \mathbf{K}_{2}^{2}, \ldots, \mathbf{K}_{2}^{n}\right) \text {, } \\
& \mathbf{K}_{2}^{i}=\frac{5 g}{7} \cdot \operatorname{diag}\left(\frac{1}{\rho_{i, 1}}, \frac{1}{\rho_{i, 2}}, \ldots, \frac{1}{\rho_{i, n_{i}}}\right) \text {, } \\
& \mathbf{K}_{1}=\left(\begin{array}{cccc}
k_{1}+k_{2} & -k_{2} & & \\
-k_{2} & k_{2}+k_{3} & -k_{3} & \\
& & \ddots & \\
& & -k_{n} & k_{n}
\end{array}\right) \text {, } \\
& \mathbf{C}_{1}=\left(\begin{array}{cccc}
c_{1}+c_{2} & -c_{2} & \\
-c_{2} & c_{2}+c_{3} & -c_{3} & \\
& & \ddots & \\
& & -c_{n} & c_{n}
\end{array}\right) \text {, }
\end{aligned}
$$

where $\operatorname{diag}($ ) denotes the diagonal matrix. The nonlinear differential equation (11) can be solved by numerical algorithms, for example, a fourth-order Runge-Kutta method.

3.3. Numerical Examples. Consider a six-floor structure with the lumped-mass model (Figure 1) subject to earthquake excitations. In the present numerical example, the external excitation in this case can be written as $F_{i}=-\left(M_{i}+\right.$ $\left.\sum_{j=1}^{n_{i}} m_{i, i}\right) \ddot{x}_{g}(t)$, where $\ddot{x}_{g}(t)$ is the earthquake acceleration, each lumped mass is assumed to be the same, at $16.32 \mathrm{t}$, the linear stiffness coefficients of each storey are 13.51, 12.86, 11.58, $9.65,7.07$, and $3.86 \times 10^{3} \mathrm{kN} / \mathrm{m}$, and the damping coefficients of each storey are selected as 27.9, 23.76, 21.39, 17.87, 13.07, and $10.04 \mathrm{kN} \cdot \mathrm{s} / \mathrm{m}$. To reduce the dynamic response of a MDOF structure efficiently, the natural frequency of the damper needs to be resonant with the structure's natural frequencies $(6.28,15.38,24.32,33.23,42.13$, and $51.02 \mathrm{rad} / \mathrm{s})$. In this respect, the natural frequency of the TRoMaDaS can be tuned to the first-mode frequency, by which the structure response is dominated. In fact, the optimum physical parameters of the tuned mass damper including the frequency and the damping coefficient, in this case relative to the rolling friction coefficient of the oscillator, are functions of the mass ratio between the main structure mass to the damper mass and other structural parameters. It can be argued that, by only tuning the frequency of the control device to the first-mode frequency of the structure, preferable control efficiency can be expected to result in the case of a low mass ratio [23]. Research on control parameter optimization-in this case, the selection of the optimum oscillator mass, rolling friction coefficient, and the radii of the arc path and the oscillator-is beyond the scope of the present paper. Invoking (10) and the first-model frequency of the structure, the radius difference between the arch paths and the oscillator can be obtained. The oscillators located in certain floors of the structure are assumed to be composed of iron with a mass density of $7800 \mathrm{~kg} / \mathrm{m}^{3}$; the rolling friction coefficients between the arch paths and the oscillators are chosen to be identical, $0.01 \mathrm{~m}$. 


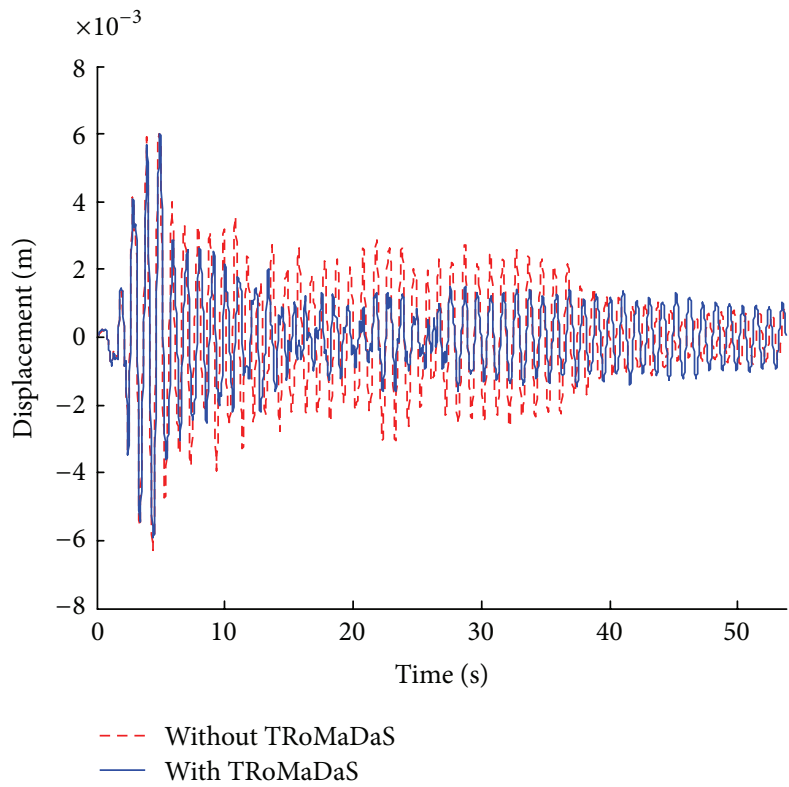

(a)

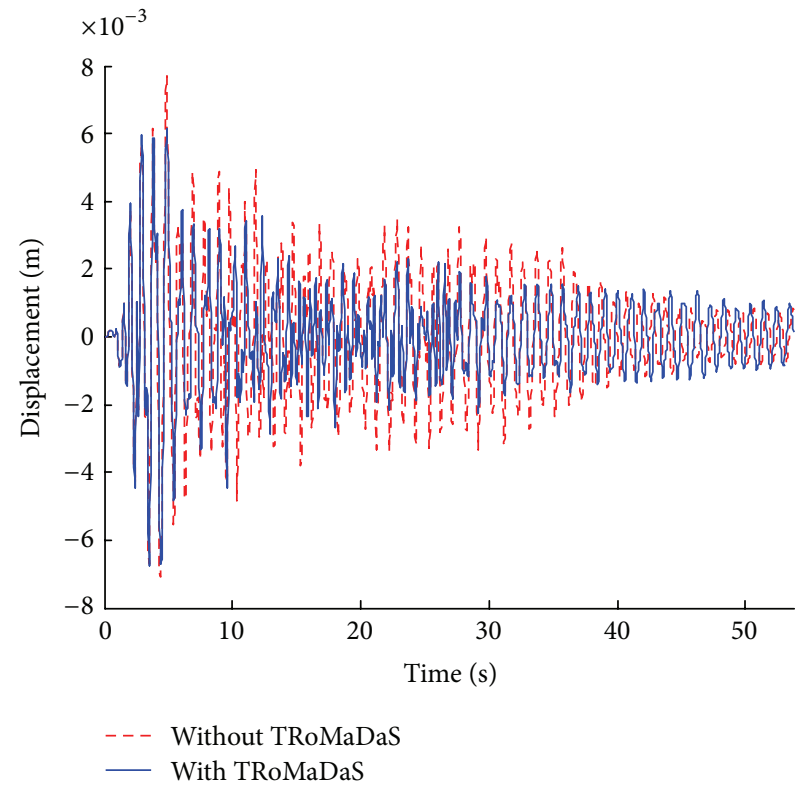

(b)

FIGURE 5: Comparison between interstorey drift ((a) first storey, (b) sixth storey) of structures with (solid line) and without (dashed line) TRoMaDaS. Structure subjected to scaled El Centro acceleration (peak acceleration $=0.513 \mathrm{~m} / \mathrm{s}^{2}$ ); TRoMaDaS oscillators with a mass ratio of $5 \%$ are distributed equally on every floor.

The mass ratio of the TRoMaDaS mass to the total mass of main structure, according to engineering considerations, could be selected to be $5 \%$. Four cases with different oscillator distributions or different levels of earthquake excitations were considered:

(a) Oscillators are equally distributed on six floors of structures subject to El Centro record NS component of the 1940 Imperial Valley earthquake (El Centro excitation for short) and JMA record NS component of the 1995 Kobe earthquake (Kobe excitation for short), with a peak value of $0.513 \mathrm{~m} / \mathrm{s}^{2}$.

(b) The damper and structure parameters are the same as in case (a), whereas the peak values of El Centro and JMA Kobe excitation increase to $1.026 \mathrm{~m} / \mathrm{s}^{2}$.

(c) The structure and excitation parameters are the same as in case (b) except that oscillators with mass ratios of $3 \%$ and $2 \%$ are installed on the third and the fourth floors, respectively.

(d) All the parameters are the same as in case (c), except that the oscillator on the fourth floor is split into 10 smaller oscillators with equal mass.

Figures 5 and 6 show the comparison of inter-storey drift in case (a) with and without the TRoMaDaS of structures subject to El Centro and Kobe excitations. From these plots, it can be seen that the peak responses (for the El Centro case, at about 3-4s, and for the Kobe case, at about 7-9s) were not reduced significantly, whereas responses during the time interval after the peak value decreased to a large degree because of the energy dissipation mechanism of the
TRoMaDaS. This phenomenon of response reduction delay can be also observed from the displacement data of a shaking table experiment of a wind turbine structure, controlled by a similar ball vibration absorber (BVA) rolling at the top of the nacelle [17]. The failure in peak response reduction may be attributable to several reasons; the most significant is the stick-slip [13] behavior of ball oscillators. The steel ball oscillators, initially resting at the bottom of the arch path, only begin to move along the paths until the acceleration of the main structure exceeds the maximum acceleration of the ball oscillator caused by the friction force/moment. That is, the TRoMaDaS can be regarded as being "activated" only when the oscillator starts rolling along the arch path after strong structure acceleration has already occurred, thus leading to a delay in response reduction and the failure of peak-value mitigation. The maximum absolute values of the interstorey drift of the controlled and uncontrolled structure and the corresponding response reduction rates, shown in Table 1, further confirm the response reduction delay effect. Also, the rotation angles of the oscillators, shown in Table 1, do not exceed the limitation of the small quantity assumption, which is often regarded as $0.3 \mathrm{rad}$ (or about ca. $20^{\circ}$ ).

The effect of the TRoMaDaS on mitigating the response during the postpeak interval may benefit the damage behavior of structures because of material fatigue. One of the widely used approaches to investigate damage behavior is based on the amount of energy absorbed by the materials. To further investigate the effectiveness of TRoMaDaS, the energy dissipation efficiency of the controlled structure was investigated. Figure 7 shows the amount of total energy input by the earthquake excitation and dissipated by the structural 
TABLE 1: Peak response of inner-storey drifts of structures with and without TRoMaDaS with an earthquake acceleration peak value of $0.513 \mathrm{~m} / \mathrm{s}^{2}$

\begin{tabular}{lccccccc}
\hline Excitation & Storey & 1 & 2 & 3 & 4 & 5 \\
\hline \multirow{4}{*}{ El Centro } & Max. uncontr. disp. (mm) & 6.29 & 6.52 & 6.81 & 7.06 & 7.13 & 7.69 \\
& Max. contr. disp. (mm) & 6.02 & 6.30 & 6.77 & 6.98 & 6.92 & 6.73 \\
& Disp. red. rate (\%) & 4.29 & 3.37 & 0.58 & 1.13 & 2.94 & 12.48 \\
& Max. ang. disp. (rad) & 0.0073 & 0.0077 & 0.0087 & 0.0268 & 0.0714 & 0.1870 \\
\hline \multirow{4}{*}{ Kobe } & Max. uncontr. (mm) & 7.35 & 7.39 & 7.35 & 7.85 & 8.78 & 10.02 \\
& Max. contr. disp. (mm) & 6.17 & 6.24 & 6.45 & 6.91 & 7.83 & 8.64 \\
& Disp. red. rate (\%) & 16.05 & 15.56 & 12.24 & 11.97 & 10.82 & 13.77 \\
& Max. ang. disp. (rad) & 0.0063 & 0.0069 & 0.0099 & 0.0180 & 0.0621 & 0.1865 \\
\hline
\end{tabular}

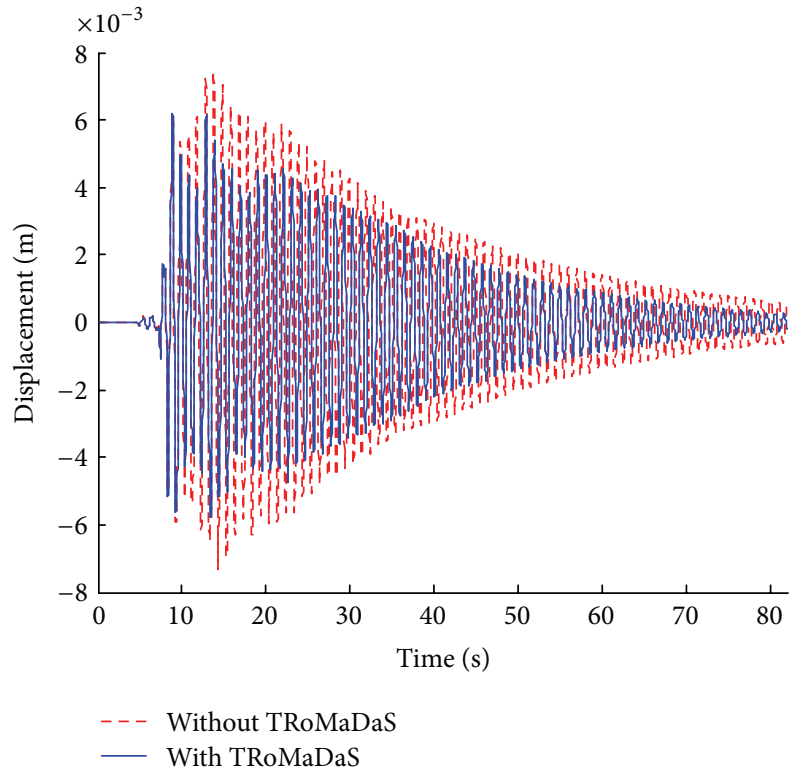

(a)

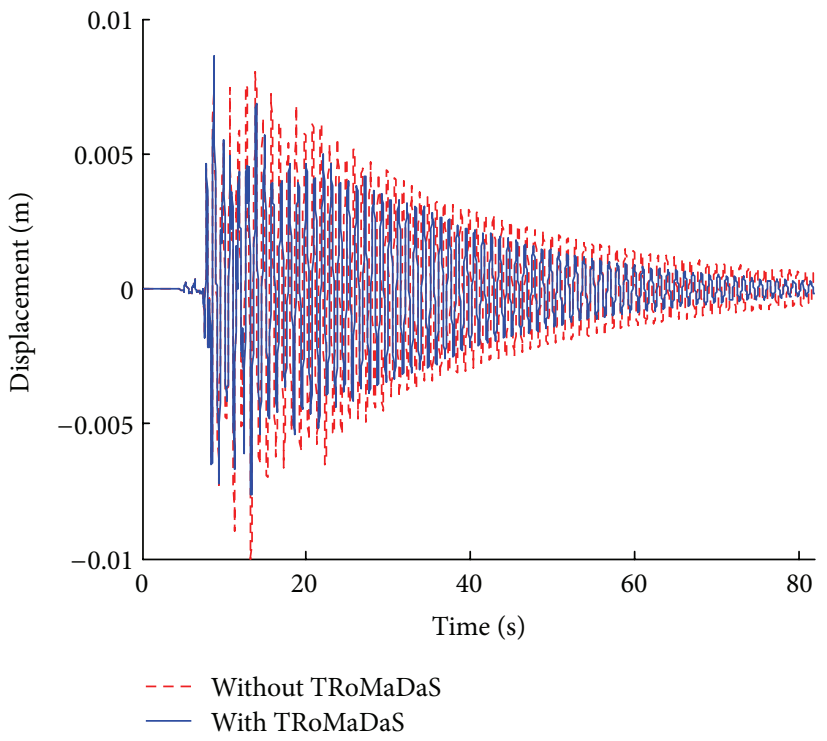

(b)

FIGURE 6: Comparison between the interstorey drift ((a) first storey, (b) sixth storey) of structures with (solid line) and without (dashed line) TRoMaDaS. Structure subjected to scaled Kobe acceleration (peak acceleration $=0.513 \mathrm{~m} / \mathrm{s}^{2}$ ); TRoMaDaS oscillators with a mass ratio of $5 \%$ are distributed equally on every floor.

damping and by the TRoMaDaS. It can be seen that, for the El Centro and Kobe excitation, respectively, about $37.5 \%$ and $40.1 \%$ of the input energy were dissipated by the TRoMaDaS.

To further investigate the response reduction efficiency of the TRoMaDaS in case (b) of intensive earthquake excitations, scaled base acceleration with a peak value $1.026 \mathrm{~m} / \mathrm{s}^{2}$ was considered. The maximum absolute interstorey drifts of the controlled and uncontrolled structures to the El Centro and Kobe excitation are shown in Table 2. However, the maximum absolute rotation angle of the oscillator in the top floor reached $1.05 \mathrm{rad}$ for the El Centro excitation and $1.01 \mathrm{rad}$ for Kobe excitation. These values, obviously, exceed the limitation of the small quantity assumption made in the derivation of the governing equations, and thus the data obtained in this situation cannot be used to assess the performance of the TRoMaDaS in reducing structure responses.

In the parameter optimization procedure of a "traditional" tuned mass damper, the stroke of the damper is an important factor that should be considered. In this situation, the stroke of the oscillator, say, the rotation angle of the ball, needs more investigation, not only from the point of view of the small quantity assumption for rotation angle, but more importantly also from the aspect of practical considerations. According to numerical investigations, the rotation angle of the oscillators can be reduced by properly locating these oscillators on certain floors. For example, in case (c), the TRoMaDaS is installed in the third and the fourth floor with $3 \%$ and $2 \%$ of the structure mass, respectively. Compared with case (b), the results of case (c) show that although the control efficiency in mitigating the peak response deteriorated, the rotation angle was reduced greatly, by about $50 \%$. The rotation angle of oscillators can be further reduced by splitting the oscillator with excess angular displacement. For example, in case (d), the oscillator in the fourth floor is split into 10 smaller ball oscillators with equal mass. In this situation, with a slight expense in terms of a deteriorated response 
TABLE 2: Peak response of inner-storey drifts of structures with and without TRoMaDaS with an earthquake acceleration peak value of $1.026 \mathrm{~m} / \mathrm{s}^{2}$

\begin{tabular}{|c|c|c|c|c|c|c|c|c|}
\hline Excitation & Storey & & 1 & 2 & 3 & 4 & 5 & 6 \\
\hline \multirow{10}{*}{ El Centro } & \multicolumn{2}{|c|}{ Max. uncontr. disp. (mm) } & 12.59 & 13.04 & 13.62 & 14.11 & 14.27 & 15.38 \\
\hline & \multirow{3}{*}{ Max. contr. disp. (mm) } & (b) & 10.36 & 10.79 & 11.46 & 11.81 & 11.56 & 13.14 \\
\hline & & (c) & 11.50 & 12.23 & 12.74 & 12.75 & 12.33 & 13.01 \\
\hline & & (d) & 12.06 & 12.79 & 13.28 & 13.24 & 12.68 & 13.00 \\
\hline & \multirow{3}{*}{ Disp. red. rate (\%) } & (b) & 17.71 & 17.25 & 15.86 & 16.30 & 18.99 & 14.56 \\
\hline & & (c) & 8.66 & 6.21 & 6.46 & 9.36 & 13.60 & 15.41 \\
\hline & & (d) & 4.21 & 1.92 & 2.50 & 6.17 & 11.14 & 15.47 \\
\hline & \multirow{3}{*}{ Max. ang. disp. (rad) } & (b) & 0.0075 & 0.0270 & 0.0993 & 0.3191 & 0.6343 & 1.0499 \\
\hline & & (c) & - & - & 0.2409 & 0.5213 & - & - \\
\hline & & (d) & - & - & 0.2730 & 0.2816 & - & - \\
\hline \multirow{10}{*}{ Kobe } & \multicolumn{2}{|c|}{ Max. uncontr. disp. (mm) } & 14.70 & 14.78 & 14.71 & 15.70 & 17.56 & 20.04 \\
\hline & \multirow{3}{*}{ Max. contr. disp. (mm) } & (b) & 12.03 & 12.25 & 12.71 & 13.59 & 15.32 & 16.36 \\
\hline & & (c) & 12.40 & 12.55 & 13.03 & 13.87 & 15.68 & 13.01 \\
\hline & & (d) & 12.45 & 12.63 & 13.11 & 13.92 & 15.67 & 13.01 \\
\hline & \multirow{3}{*}{ Disp. red. rate (\%) } & (b) & 18.16 & 17.12 & 13.60 & 13.44 & 12.76 & 18.36 \\
\hline & & (c) & 15.65 & 15.09 & 11.42 & 11.66 & 10.71 & 35.08 \\
\hline & & (d) & 15.31 & 14.55 & 10.88 & 11.34 & 10.76 & 35.08 \\
\hline & \multirow{3}{*}{ Max. ang. disp. (rad) } & (b) & 0.0061 & 0.0309 & 0.1199 & 0.3082 & 0.6185 & 1.0127 \\
\hline & & (c) & - & - & 0.2216 & 0.5352 & - & - \\
\hline & & (d) & - & - & 0.2618 & 0.2593 & - & - \\
\hline
\end{tabular}

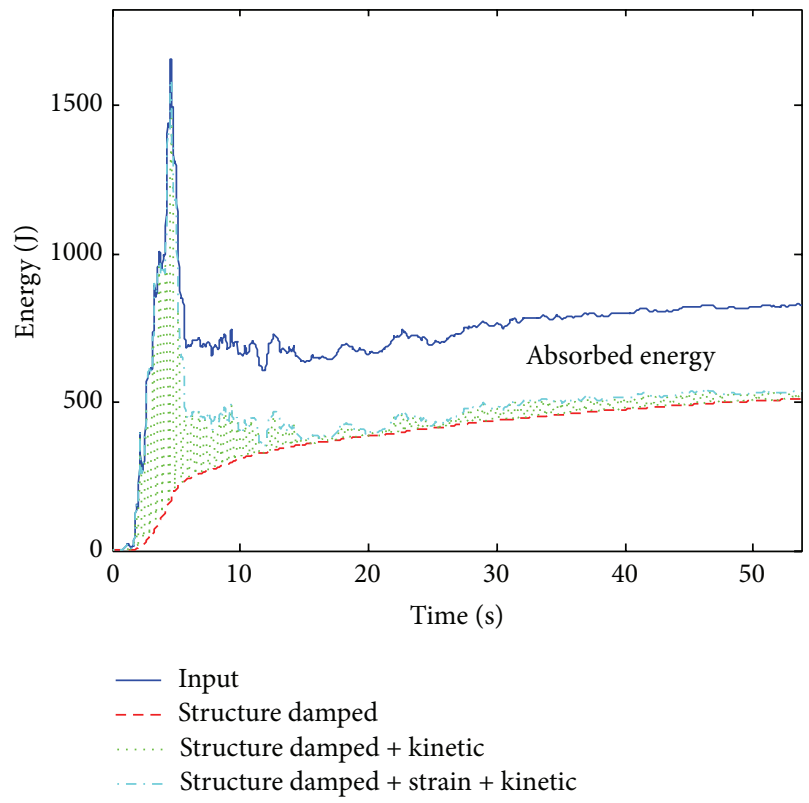

(a)

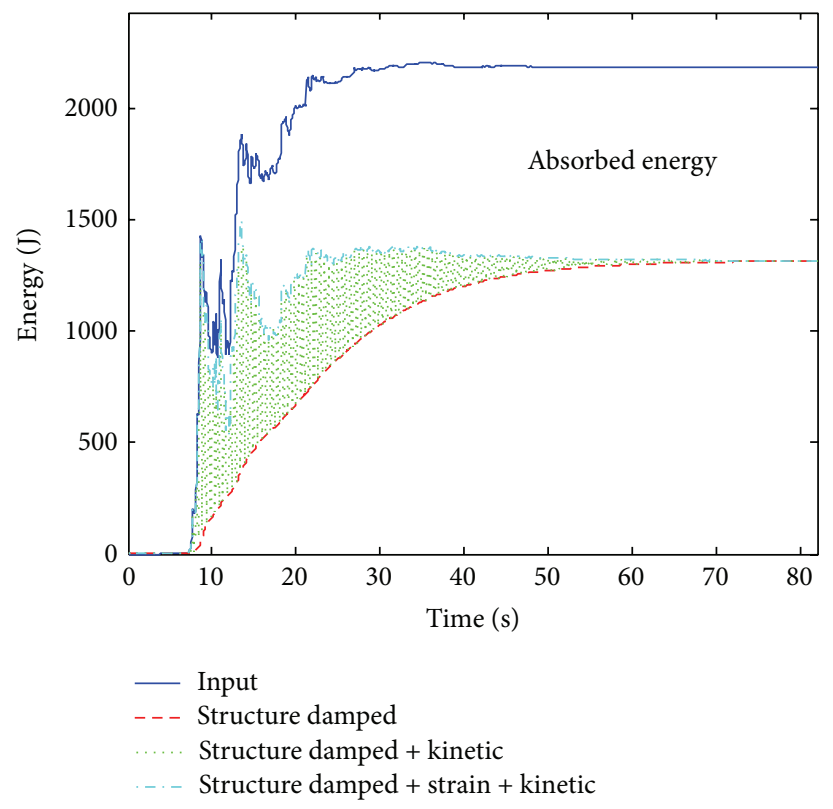

(b)

FIGURE 7: Cumulative energy time history for TRoMaDaS-damped MDOF structure. Structure subjected to scaled El Centro (shown in (a) with peak acceleration $=0.513 \mathrm{~m} / \mathrm{s}^{2}$ ) and Kobe ( mass ratio of $5 \%$ are distributed equally on every floor.

reduction, the angular displacement is greatly reduced, below the limitations of the small quantity assumption. Figures 8 and 9 show a comparison of interstorey drift of the first and the top floor with and without the TRoMaDaS in case (d). Figure 10 shows the efficiency of the TRoMaDaS in case (d) from the energy perspective. From these plots, it was further confirmed that, for both the El Centro and Kobe excitation, the peak values were not mitigated significantly, whereas over $50 \%$ of the input seismic energy was dissipated by the proposed TRoMaDaS. 


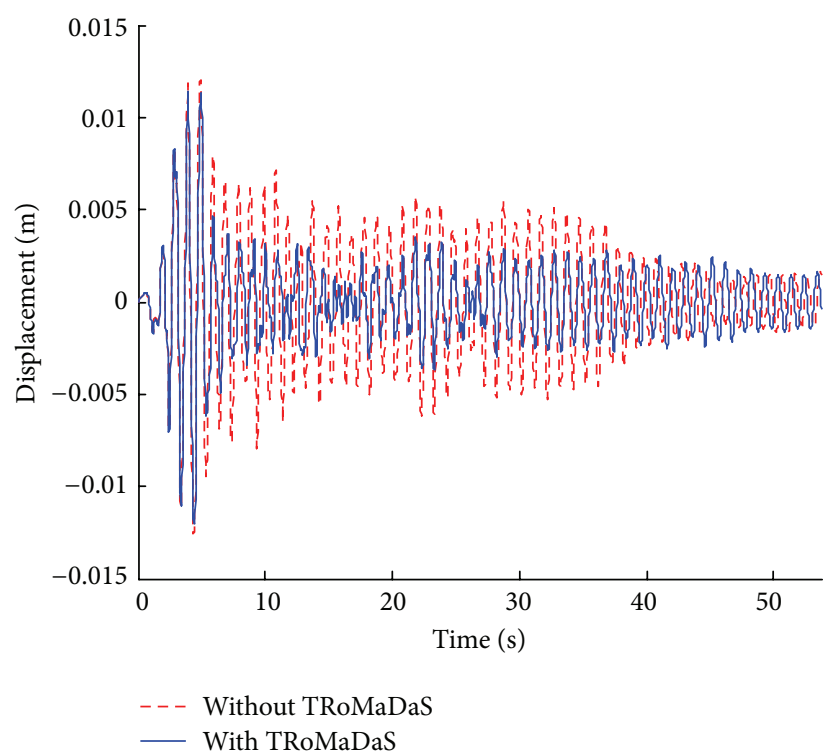

(a)

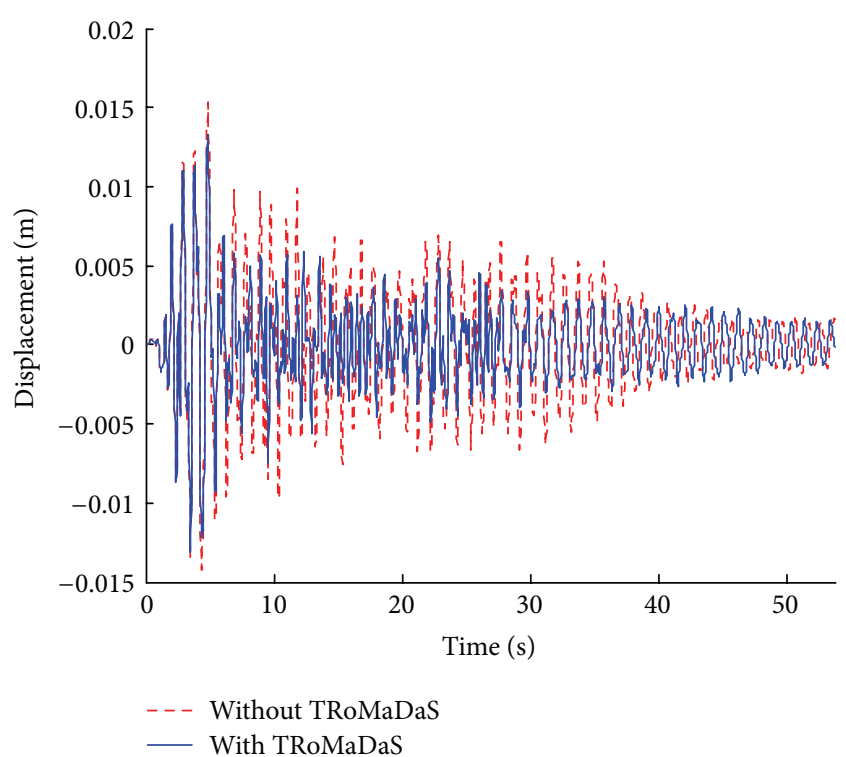

(b)

FIGURE 8: Comparison between interstorey drift ((a) first storey, (b) sixth storey) of structures with (solid line) and without (dashed line) TRoMaDaS. Structure subjected to scaled El Centro acceleration (peak acceleration $=1.026 \mathrm{~m} / \mathrm{s}^{2}$ ). TRoMaDaS oscillators with a mass ratio of $5 \%$ are installed on the third (one ball) and fourth floors (10 balls with equal mass) of $3 \%$ and $2 \%$ of the main structure mass, respectively.

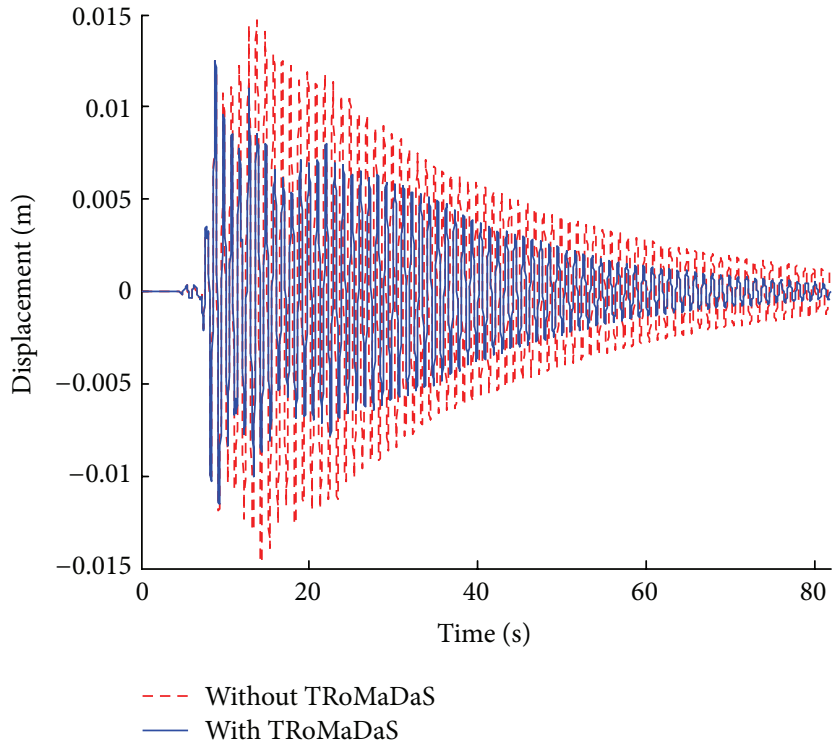

(a)

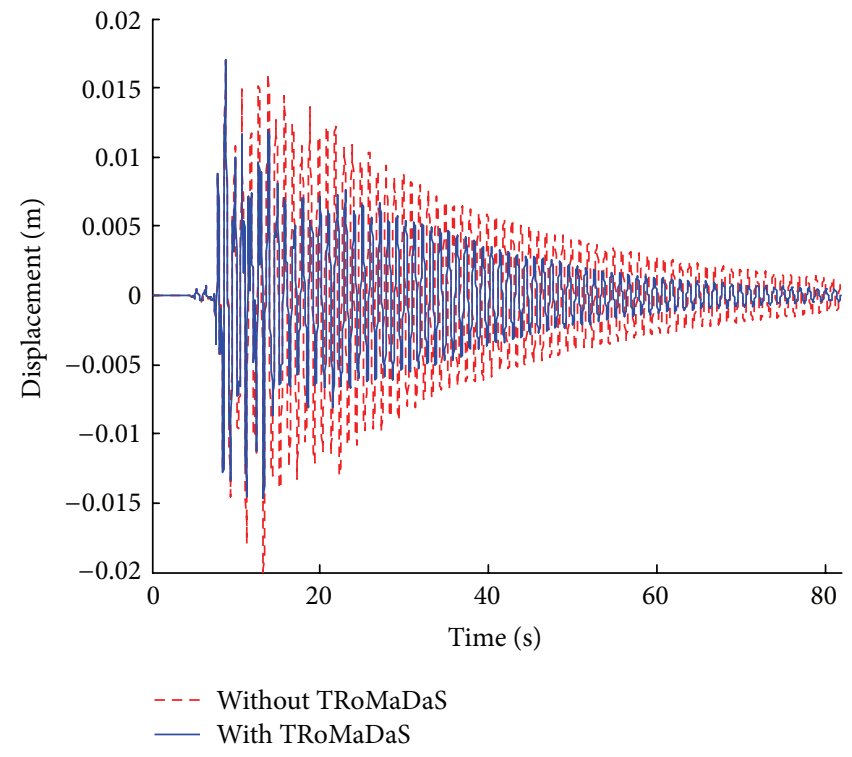

(b)

FIGURE 9: Comparison between the interstorey drift ((a) first storey, (b) sixth storey) of structures with (solid line) and without (dashed line) TRoMaDaS. Structure subject to scaled Kobe acceleration (peak acceleration $=1.026 \mathrm{~m} / \mathrm{s}^{2}$ ). TRoMaDaS oscillators are installed on the third (one ball) and the fourth floor (10 balls with equal mass) of $3 \%$ and $2 \%$ of the main structure mass, respectively.

\section{Stochastic Dynamic Analysis}

It can be seen from the numerical analysis above that, because of the uncertain input of the earthquake excitation, the control efficiency of the TRoMaDaS appears to differ for different excitations even with the same intensity (e.g., compare case (d) with the El Centro and Kobe excitations). Using the nonlinear random vibration theory, one can investigate the control efficiency of the proposed device in a different, probabilistic manner. Furthermore, the optimization of control parameters in future investigations should be implemented similarly, based on a stochastic perspective.

Stochastic dynamic analysis as an engineering application was begun in 1958 by Crandall [24] for mechanical engineering. This theory was soon used to investigate the random responses of civil structures to quantify the uncertainty 


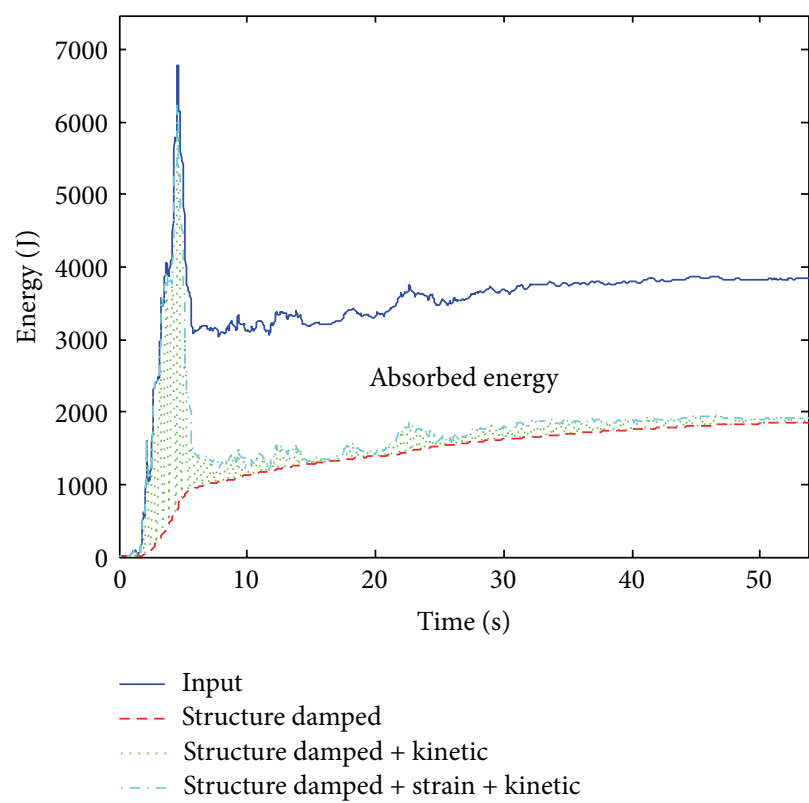

(a) El Centro excitation

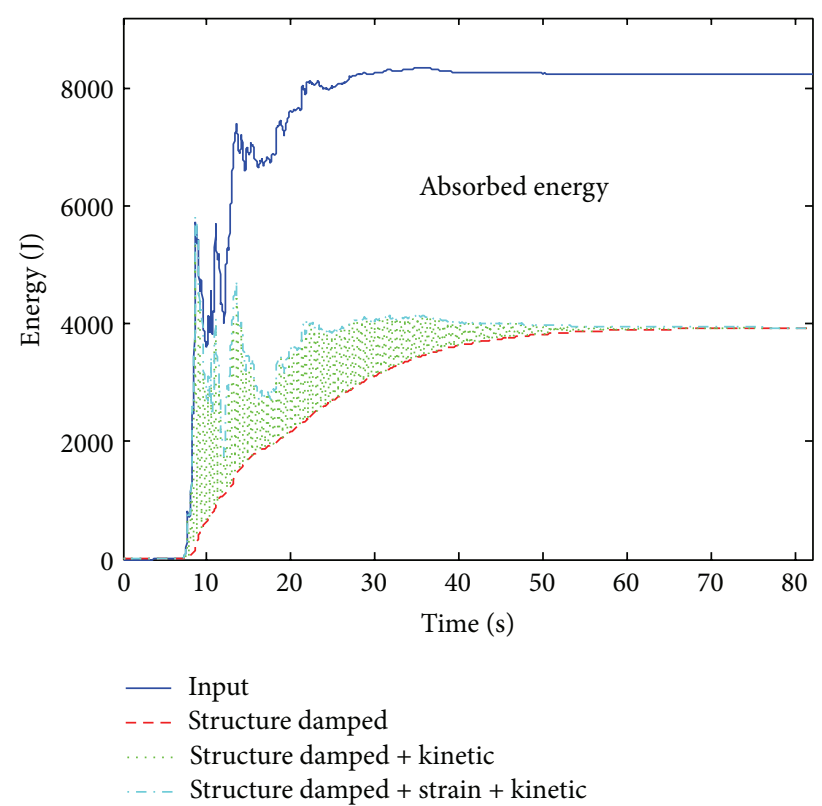

(b) Kobe excitation

FIGURE 10: Cumulative energy time history for TRoMaDaS-damped MDOF structure. Structure subjected to scaled El Centro (shown in (a) with peak acceleration $=1.026 \mathrm{~m} / \mathrm{s}^{2}$ ) and Kobe (shown in $(\mathrm{b})$ with peak acceleration $=1.026 \mathrm{~m} / \mathrm{s}^{2}$ ) acceleration; TRoMaDaS oscillators are installed in the third (one ball) and the fourth floor (10 balls with equal mass) of $3 \%$ and $2 \%$ of the main structure mass, respectively.

propagation of ambient stochastic excitations. Later, stochastic methods considering nonlinear and even hysteretic behavior in civil structures were developed by researchers. These methods include stochastic averaging [25], statistical linearization/nonlinearization [26], moment closure [27], and Monte Carlo simulations [28]. Among them, the statistical linearization and Monte Carlo methods are two that are used widely, although the latter often suffers from computational inefficiency problems, especially for large-scale civil structures. In this section, to consider the uncertainty propagation and to further extend the stochastic analysis of a TRoMaDaScontrolled MDOF structure, statistical linearization was used to determine the standard deviation of displacement, in a closed form, of a SDOF structure with a controlling device.

\subsection{Analytical Solution for SDOF Structure with TRoMaDaS.} For simplicity and for the purposes of this preliminary investigation of the stochastic control efficiency of the proposed control system, a single-degree-of-freedom (SDOF) structural system with a single oscillator ball subject to white noise excitation was considered. The stochastic differential equation of motion of this system can be written as

$$
\mathbf{M Z}+\mathbf{C Z}+\mathbf{K Z}+\mathbf{f}(\mathbf{Z}, \dot{\mathbf{Z}})=\mathbf{w}(t),
$$

where $\mathbf{Z}$ is the stochastic response and

$$
\mathbf{M}=\left[\begin{array}{cc}
M+m & m \rho \\
\frac{5}{7 \rho} & 1
\end{array}\right],
$$

$$
\begin{aligned}
& \mathbf{C}=\left[\begin{array}{ll}
c & 0 \\
0 & 0
\end{array}\right], \\
& \mathbf{K}=\left[\begin{array}{cc}
k & 0 \\
0 & \frac{5 g}{7 \rho}
\end{array}\right], \\
& \mathbf{w}=\left\{\begin{array}{c}
w(t) \\
0
\end{array}\right\},
\end{aligned}
$$

where $w(t)$ is the Gaussian white noise excitation with power spectrum $S_{0}$. The linearized equation of motion of the structure with a TRoMaDaS (15) can be written in the form

$$
\mathbf{M} \ddot{\mathbf{Z}}(t)+\left(\mathbf{C}+\mathbf{C}_{e}\right) \dot{\mathbf{Z}}(t)+\mathbf{K Z}(t)=-\mathbf{w}(t),
$$

where $\mathbf{Z}=(X, \Theta)^{T}$ is the augmented displacement vector and $\mathbf{C}_{e}$ is the additional equivalent damping matrix that can be appropriately determined by minimizing the difference between (15) and (17) in the mean squared sense (see [26] for details). In this regard, for a chain-like controlled structural systems, $\mathbf{C}_{e}$ can be determined by

$$
c_{i, j}^{e}=E\left\{\frac{\partial f_{i}}{\partial \dot{x}_{j}}\right\}, \quad i=1,2 ; j=1,2,
$$


where $\mathbf{f}(\mathbf{Z}, \dot{\mathbf{Z}})=\left(0,5 \mu g R /\left(7 \rho^{2} r\right) \operatorname{sgn}(\dot{\Theta})\right)^{T}$ is the nonlinear damping in (15). Furthermore, according to (18),

$$
\begin{aligned}
C_{\mathrm{eq}} & =\left[\begin{array}{cc}
0 & 0 \\
0 & c_{22}^{\mathrm{eq}}
\end{array}\right], \\
c_{22}^{\mathrm{eq}} & =\beta_{\mathrm{eq}}=\frac{5 \mu g R}{7 r \rho^{2} \sigma_{\Theta}} \sqrt{\frac{2}{\pi}},
\end{aligned}
$$

where $\sigma_{\dot{\Theta}}$ is the standard deviation of the angular velocity of the oscillator. Furthermore, combining with (10) yields the equivalent damping ratio of the nonlinear equation of motion of the oscillator as

$$
\zeta_{\mathrm{eq}}=\frac{\beta_{\mathrm{eq}}}{2 \omega_{0}}=\sqrt{\frac{5 g}{14 \pi \rho^{3}}} \frac{\mu R}{r \sigma_{\dot{\Theta}}},
$$

where $\omega_{0}$ is the natural frequency of the oscillator.

Next, the standard deviation of the response of an equivalent linear system can be determined according to linear random vibration theory; that is,

$$
\boldsymbol{\sigma}_{\dot{\mathbf{X}}}=\int_{-\infty}^{\infty} \mathbf{S}_{\dot{\mathbf{X}} \dot{\mathbf{X}}}(\omega) \mathrm{d} \omega=\int_{-\infty}^{\infty} \omega^{2} \mathbf{S}_{\mathbf{X X}}(\omega) \mathrm{d} \omega,
$$

where $\mathbf{S}_{\dot{\mathbf{x}} \dot{\mathbf{x}}}(\omega)$ is the power spectrum density matrix of the system displacement that can be determined by

$$
\mathbf{S}_{\mathbf{X X}}(\omega)=\mathbf{H}^{T}(\omega) \mathbf{S}_{\mathbf{F F}}(\omega) \mathbf{H}(\omega)
$$

with $\mathrm{S}_{\mathrm{FF}}(\omega)$ being the power spectrum density matrix of the excitation $\mathbf{w}(t)=(w(t), 0)$. Clearly, because of the special form of the excitation vector, in the matrix $\mathbf{H}(\omega)$, only $H_{11}(\omega)$ and $H_{21}(\omega)$ are needed for the autospectrum $S_{X X}(\omega)$ and $S_{\Theta \Theta}(\omega)$. In this regard, these frequency transfer functions can be solved by

$$
\mathbf{M}(\mathbf{p} ; \omega) \mathbf{h}(\omega)=\mathbf{N}
$$

where

$$
\begin{aligned}
& \mathbf{M}(\mathbf{p} ; \omega) \\
& =\left[\begin{array}{cc}
-\omega^{2}(M+m)+i \omega c+k & -\omega^{2} m p \\
-\frac{5 \omega^{2}}{7 \rho} & -\omega^{2}+i \omega \beta_{\mathrm{eq}}+\frac{5 g}{7 \rho}
\end{array}\right], \\
& \mathbf{h}(\omega)=\left\{\begin{array}{l}
H_{11}(\omega) \\
H_{21}(\omega)
\end{array}\right\}, \\
& \mathbf{N}=\left\{\begin{array}{l}
1 \\
0
\end{array}\right\},
\end{aligned}
$$

where $p=\left(M, m, c, k, \rho, \beta_{\text {eq }}\right)$ is parameters of equivalent linear systems.

At this point, it can be argued that (20) and (22)-(24) constitute a mutually dependent relationship between $\sigma_{\dot{\Theta}}$ and equivalent damping coefficient that can be solved in an iterative manner. However, (22) is always difficult to obtain in an explicit closed form although numerical algorithms can be used to obtain the relationship in a discrete form. In this regard, a closed-form integration approach was developed by Roberts and Spanos [26], providing an accurate method for determining the standard deviation from the power spectrum density (see the Appendix for the standard deviation of the responses in detail).

4.2. Numerical Example. Consider a SDOF structure with the lumped mass being $8 \times 10^{3} \mathrm{~kg}$. The stiffness and the damping ratio are $2 \times 10^{5} \mathrm{~N} / \mathrm{m}$ and 0.02 , respectively. The structure is initially at rest and subject to stochastic earthquake excitation, modeled by a zero-mean Gaussian white noise with the power spectrum strength being $3 \times 10^{-3} \mathrm{~m} \cdot \mathrm{s}^{-3}$. The natural frequency of the structure is $\omega_{0}=5 \mathrm{rad} / \mathrm{s}$ and the ratio between the mass of the oscillator ball and the primary structure is selected to be $5 \%$. To obtain preferable control efficiency, the natural frequency of the oscillator ball is tuned to agree with the one of the structure. In this regard, the radii of the oscillator ball and of the arc path are $r=0.337 \mathrm{~m}$ and $R=r+\rho=0.617$, where $\rho=0.280 \mathrm{~m}$. According to (10), the radius difference between these two radii is a factor on which the natural frequency of the oscillator depends.

To validate the randomness in the control efficiency, a comparison of the response displacements between uncontrolled and controlled structures subjected to two different sample excitations is plotted in Figure 11. From these plots, it can be concluded that the structure response is not mitigated efficiently until the response surpasses a certain level. This is probably, according to the analysis in Section 3.3, due to the stick-slip behavior of the TRoMaDaS. Moreover, it can be seen that the "rear" part of the response displacement of the controlled structure, shown in Figures 11(a) and 11(b), behaves differently, confirming the need for a probabilistic investigation of the control efficiency. Figure 12 shows a comparison of the standard deviation of the response displacement between the structures with and without TRoMaDaS. It can be seen from this figure that not only is the displacement standard deviation reduced to a large extent but also that the stochastic response of the structure readily becomes stationary because of the TRoMaDaS.

\section{Concluding Remarks}

A novel tuned mass damper system for civil structures named tuned rolling mass damper system or TRoMaDaS has been proposed in this paper. Compared with the traditional installation of tuned mass dampers, the most promising feature of the proposed passive control system is that it does not require a dedicated space to accommodate the oscillator and thus maintains the integrity of civil buildings. To investigate the seismic response reduction of the proposed passive control system, the equation of motion of the controlled system subject to external dynamic excitation was derived. In this regard, a physical model of the passively controlled structure was modeled as a chain-like MDOF system with arbitrary number of oscillators rolling within the hollow floors. Lagrange's equation was used to derive the equation of motion of 


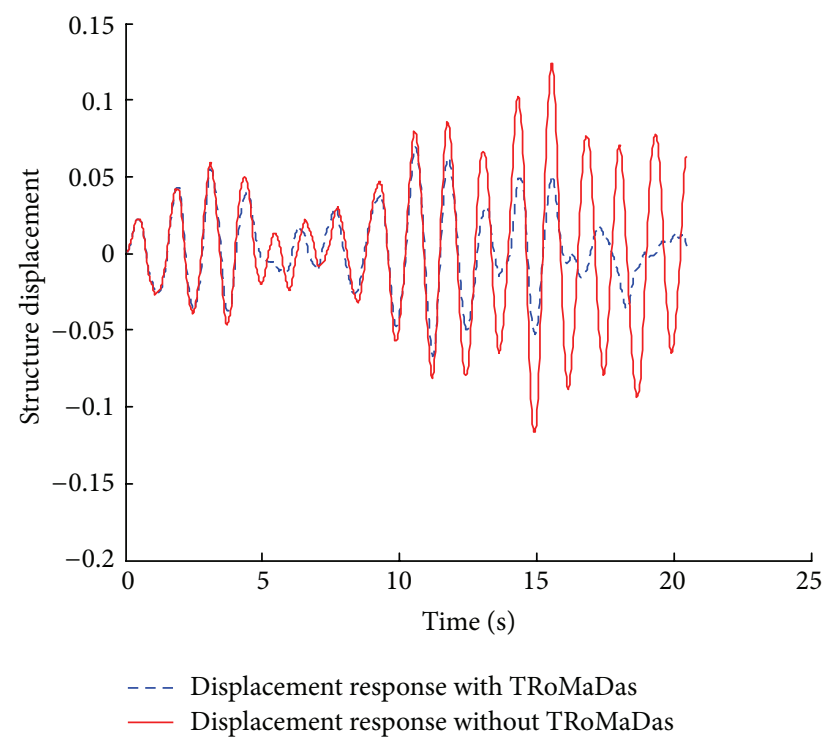

(a)

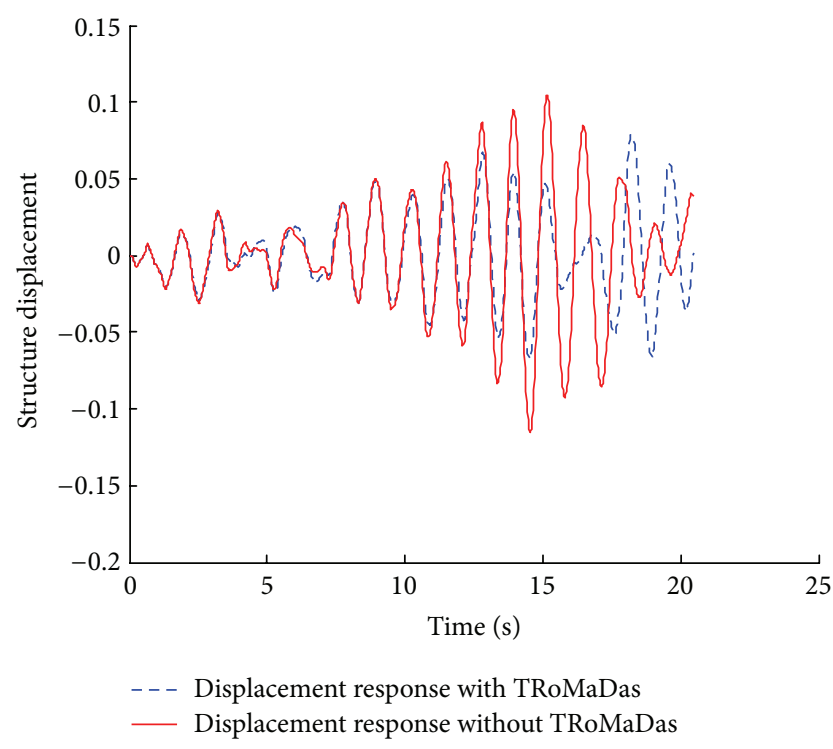

(b)

Figure 11: Comparison of displacement responses between controlled (dashed line) and uncontrolled (solid line) structures subject to different samples ((a) first sample, and (b) second sample) of Gaussian white noise with power spectrum strength $S_{0}=3 \times 10^{-3} \mathrm{~m} \cdot \mathrm{s}^{-3}$. The frequency of the oscillator balls with a $5 \%$ mass ratio is tuned to the frequency of the main structure.

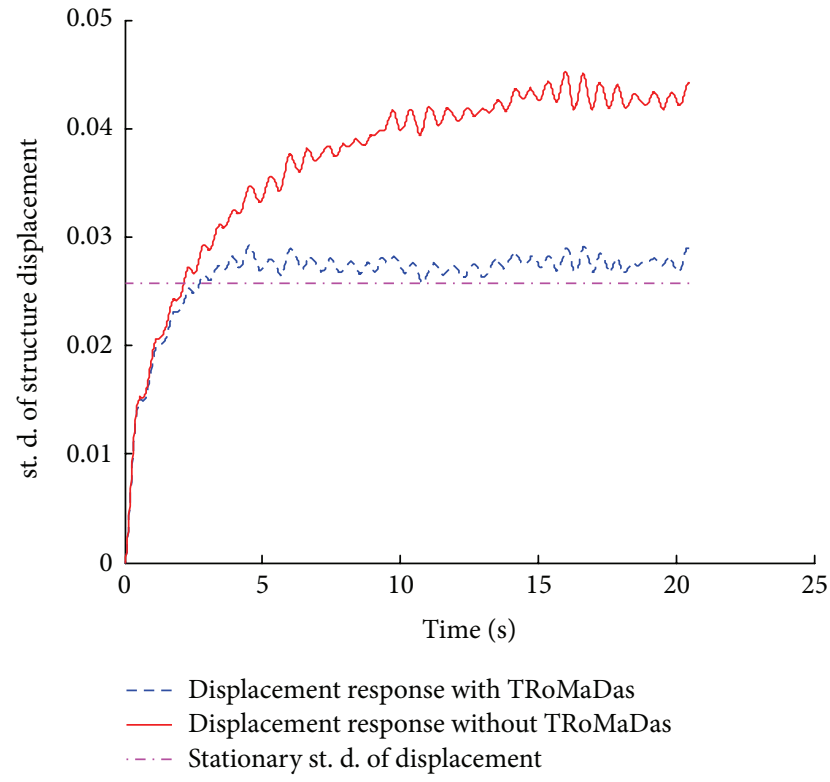

Figure 12: Comparison of the standard deviation (st. d.) of the response displacements between the uncontrolled structure, calculated by a Monte Carlo simulation (solid line), and the controlled structure, calculated by a Monte Carlo simulation (dashed line), and a statistical linearization method (dash-dot line).

the complex coupled nonlinear dynamic system. Numerical methods such as the fourth-order Runge-Kutta method were used to solve the dynamic equation to investigate the control efficiency of the TRoMaDaS. Several cases with different TRoMaDaS distributions and different numbers of oscillators were studied.
It can be concluded that, because of the so-called "stickslip" behavior of friction-type TMDs, although the peak response cannot be mitigated efficiently, a large amount of seismic energy input is dissipated by the TRoMaDaS. This promising feature of TRoMaDaS allows its installation in structures where fatigue is the leading cause of damage. Furthermore, it was found that the location/distribution of the TRoMaDaS influences not only the response reduction of the controlled structure, but also the angular displacement of the oscillators. Moreover, an increased number of oscillators located on a certain floor have the effect of decreasing their angular displacement, providing an alternative means of limiting the stroke of the oscillator. Finally, the statistical linearization method was used to investigate the control efficiency of the proposed control system in a probabilistic manner. It was found that not only was the displacement standard deviation reduced by a large extent but also the stochastic response of the structure readily became stationary because of the TRoMaDaS.

The investigations detailed in this paper on the potential structural control capability of the proposed TRoMaDaS are preliminary. Further studies should focus on investigations regarding the three-dimensional motion of the oscillator, torsional control capacity of irregular structures, releasing of the small-quantity assumption for oscillators, and optimum control parameter selection for the proposed TRoMaDaS.

\section{Appendix}

From (22)-(23), it can be seen that the standard deviation of the stationary response can be written in integral form as

$$
I_{m}=\int_{-\infty}^{\infty} \frac{\Xi_{m}(\omega)}{\Lambda_{m}(-i \omega) \Lambda_{m}(i \omega)} \mathrm{d} \omega
$$


where

$$
\begin{gathered}
\Xi_{m}(\omega)=\xi_{m-1} \omega^{2 m-2}+\xi_{m-2} \omega^{2 m-4}+\cdots+\xi_{0}, \\
\Lambda_{m}(\omega)=\lambda_{m}(i \omega)^{m}+\lambda_{m-1}(i \omega)^{m-1}+\cdots+\lambda_{0} .
\end{gathered}
$$

Equation (A.1) can be further calculated by division of two determinants involving coefficients in (A.2); that is,

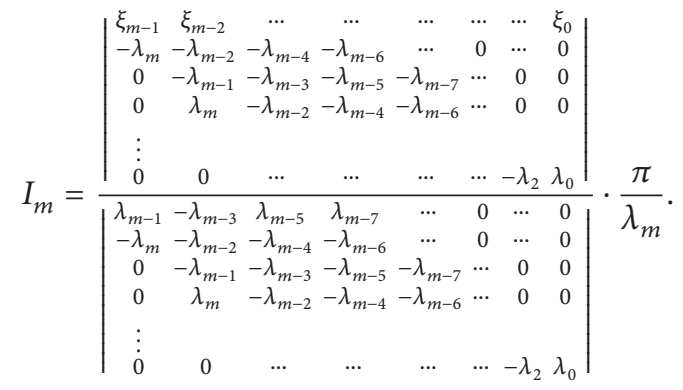

Specifically, the standard deviation of the rotational angular displacement of the oscillator with respect to the center of the arc path can be calculated as

$$
\sigma_{\Theta}^{2}=\int_{-\infty}^{\infty} S_{\Theta \Theta}(\omega) \mathrm{d} \omega=\int_{-\infty}^{\infty} S_{0}\left|H_{21}(\omega)\right|^{2} \mathrm{~d} \omega
$$

which can be calculated with (A.3) and

$$
\begin{aligned}
& \lambda_{4}=M+\frac{2 m}{7}, \\
& \lambda_{3}=c+(M+m) \beta_{\mathrm{eq}}, \\
& \lambda_{2}=k+\frac{c \beta_{\mathrm{eq}} 5 q(M+m)}{7 \rho}, \\
& \lambda_{1}=\beta_{\mathrm{eq}} k+\frac{5 c g}{7 \rho} .
\end{aligned}
$$

The standard deviation of the rotational angular velocity of the oscillator with respect to the center of the arc path can be calculated as

$$
\sigma_{\dot{\Theta}}^{2}=\int_{-\infty}^{\infty} S_{\dot{\Theta} \dot{\Theta}}(\omega) \mathrm{d} \omega=\int_{-\infty}^{\infty} S_{0} \omega^{2}\left|H_{21}(\omega)\right|^{2} \mathrm{~d} \omega
$$

which can be also solved with (A.3) and $\xi_{3}=1, \xi_{2}=\xi_{1}=\xi_{0}=$ 0 , and $\lambda_{i}, i=1,2,3,4$, as shown in (A.5a), (A.5b), (A.5c), and (A.5d).

The standard deviation of the primary structure displacement thus can be determined as

$$
\sigma_{X}^{2}=\int_{-\infty}^{\infty} S_{X X}(\omega) \mathrm{d} \omega=\int_{-\infty}^{\infty} S_{0}\left|H_{11}(\omega)\right| \mathrm{d} \omega
$$

with $\xi_{3}=0, \xi_{2}=1, \xi_{1}=-10 g /(7 \rho)+\beta_{\mathrm{eq}}, \xi_{0}=25 g^{2} /\left(49 \rho^{2}\right)$, and $\lambda_{i}, i=1,2,3,4$, as shown in (A.5a), (A.5b), (A.5c), and (A.5d).

\section{Disclosure}

The English in this document has been checked by at least two professional editors, both native speakers of English.

\section{Conflict of Interests}

The authors declare that there is no conflict of interests regarding the publication of this paper.

\section{Acknowledgments}

This work was supported financially by the National Natural Science Foundation of China (NSFC) (Grant no. 51408451), the Natural Science Foundation of Hubei Province (Grant no. 2014CFB841), and the Fundamental Research Funds for the Central Universities of China (Grant no. WUT-2014-IV-051).

\section{References}

[1] J. T. P. Yao, "Concept of structural control," Journal of Structural Division, vol. 98, no. 7, pp. 1567-1574, 1972.

[2] T. T. Soong and G. F. Dargush, Passive Energy Dissipation Systems in Structural Engineering, John Wiley \& Sons, Chichester, UK, 1997.

[3] G. W. Housner, L. A. Bergman, T. K. Caughey et al., "Structural control: past, present, and future," Journal of Engineering Mechanics, vol. 123, no. 9, pp. 897-971, 1997.

[4] K. C. S. Kwok and B. Samali, "Performance of tuned mass dampers under wind loads," Engineering Structures, vol. 17, no. 9, pp. 655-667, 1995.

[5] Y. Tamura, "Suppression of wind-induced vibrations of buildings," Journal of Wind Engineering, vol. 1990, no. 44, pp. 71-84, 1990.

[6] T. Lai, Structural behavior of BubbleDeck slabs and their application to lightweight bridge decks [M.S. thesis], Massachusetts Institute of Technology, Cambridge, Mass, USA, 2009.

[7] A. Churakov, "Biaxial hollow slab with innovative types of voids," Construction of Unique Buildings \& Structures, vol. 6, pp. 70-88, 2014.

[8] B.-D. Li, J.-J. Li, and L.-M. Fu, "Research on the bonding properties of case-in-place reinforced concrete dense rib cavity ceiling," Journal of Wuhan University of Technology, vol. 31, no. 10, pp. 44-47, 2009.

[9] M. Schnellenbach-Held and K. Pfeffer, "Punching behavior of biaxial hollow slabs," Cement and Concrete Composites, vol. 24, no. 6, pp. 551-556, 2002.

[10] W. B. Ali and G. S. Urgessa, "Structural capacities of spherically voided biaxial slab," in Structures Congress, pp. 785-796, Boston, Mass, USA.

[11] M. Pirner, "Actual behaviour of a ball vibration absorber," Journal of Wind Engineering and Industrial Aerodynamics, vol. 90, no. 8, pp. 987-1005, 2002.

[12] J. Náprstek, C. Fischer, M. Pirner, and O. Fischer, "Non-linear model of a ball vibration absorber," in Computational Methods in Earthquake Engineering, vol. 30 of Computational Methods in Applied Sciences, pp. 381-396, Springer, Dordrecht, The Netherlands, 2013.

[13] F. Ricciardelli and B. J. Vickery, "Tuned vibration absorbers with dry friction damping," Earthquake Engineering \& Structural Dynamics, vol. 28, no. 7, pp. 707-723, 1999.

[14] K. Kitamura, T. Ohkuma, J. Kanda, Y. Mataki, and S. Kawabata, "Chiba Port Tower: full-scale meaurement of wind actions (part I): organisation, measurement system and strong wind data," Journal of Wind Engineering, vol. 37, pp. 401-410, 1988. 
[15] K. Ohtake, Y. Mataki, T. Ohkuma, J. Kanda, and H. Kitamura, "Full-scale measurements of wind actions on Chiba Port Tower," Journal of Wind Engineering and Industrial Aerodynamics, vol. 43, no. 1-3, pp. 2225-2236, 1992.

[16] M. Pirner, "Dissipation of kinetic energy of large-span bridges," Acta Technica CSAV, vol. 39, pp. 645-655, 1994.

[17] Z.-L. Zhang, J.-B. Chen, and J. Li, "Theoretical study and experimental verification of vibration control of offshore wind turbines by a ball vibration absorber," Structure and Infrastructure Engineering: Maintenance, Management, Life-Cycle Design and Performance, vol. 10, no. 8, pp. 1087-1100, 2013.

[18] J. L. Chen and C. T. Georgakis, "Tuned rolling-ball dampers for vibration control in wind turbines," Journal of Sound and Vibration, vol. 332, no. 21, pp. 5271-5282, 2013.

[19] O. Fisher and M. Pirner, "The ball absorber-a new tool for passive energy dissipation of vibrations of high buildings," in Proceedings of the 7th International Seminar on Seismic Isolation, Passive Energy Dissipation and Active Control of Vibrations of Structures, pp. 103-110, Assisi, Italy, 2002.

[20] E. Matta, A. De Stefano, and B. F. J. Spencer, "A new passive rolling-pendulum vibration absorber using a non-axialsymmetrical guide to achieve bidirectional tuning," Earthquake Engineering \& Structural Dynamics, vol. 38, no. 15, pp. 17291750, 2009.

[21] M. P. Singh, S. Singh, and L. M. Moreschi, "Tuned mass dampers for response control of torsional buildings," Earthquake Engineering \& Structural Dynamics, vol. 31, no. 4, pp. 749-769, 2002.

[22] R. S. Jangid and T. K. Datta, "Performance of multiple tuned mass dampers for torsionally coupled system," Earthquake Engineering \& Structural Dynamics, vol. 26, no. 3, pp. 307-317, 1997.

[23] F. Rüdinger, "Tuned mass damper with nonlinear viscous damping," Journal of Sound and Vibration, vol. 300, no. 3-5, pp. 932-948, 2007.

[24] S. H. Crandall, Random Vibration, MIT Press, Cambridge, Mass, USA, 1958.

[25] J. B. Roberts and P. D. Spanos, "Stochastic averaging: an approximate method of solving random vibration problems," International Journal of Non-Linear Mechanics, vol. 21, no. 2, pp. $111-134,1986$.

[26] J. B. Roberts and P. D. Spanos, Random Vibration and Statistical Linearization, Dover, New York, NY, USA, 2003.

[27] S. H. Crandall, "Non-gaussian closure for random vibration of non-linear oscillators," International Journal of Non-Linear Mechanics, vol. 15, no. 4-5, pp. 303-313, 1980.

[28] P. D. Spanos and B. A. Zeldin, "Monte Carlo treatment of random fields: a broad perspective," Applied Mechanics Reviews, vol. 51, no. 3, pp. 219-237, 1998. 

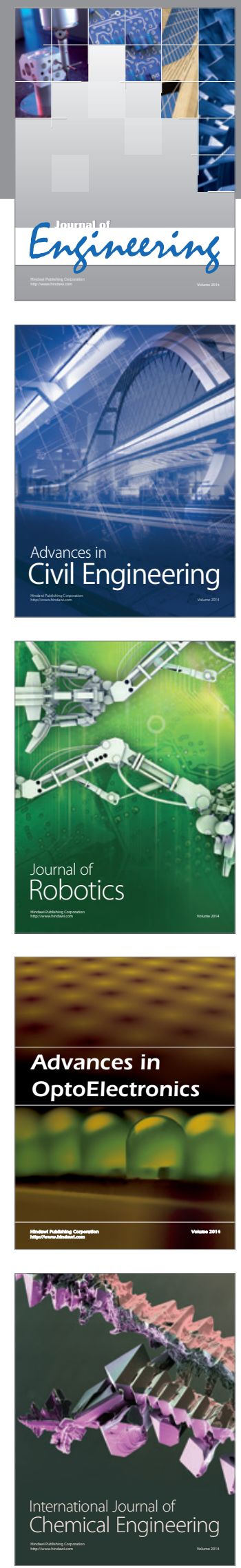

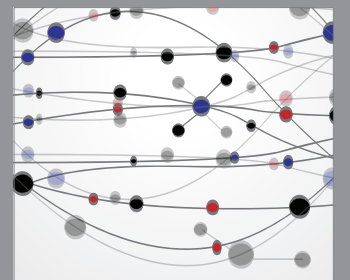

The Scientific World Journal
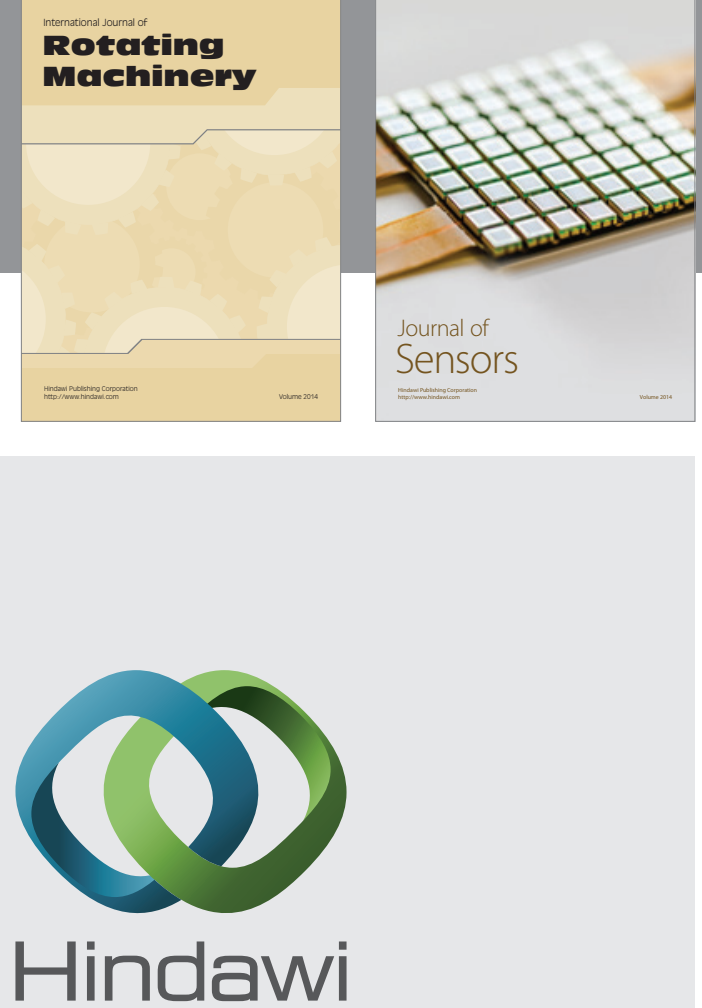

Submit your manuscripts at http://www.hindawi.com
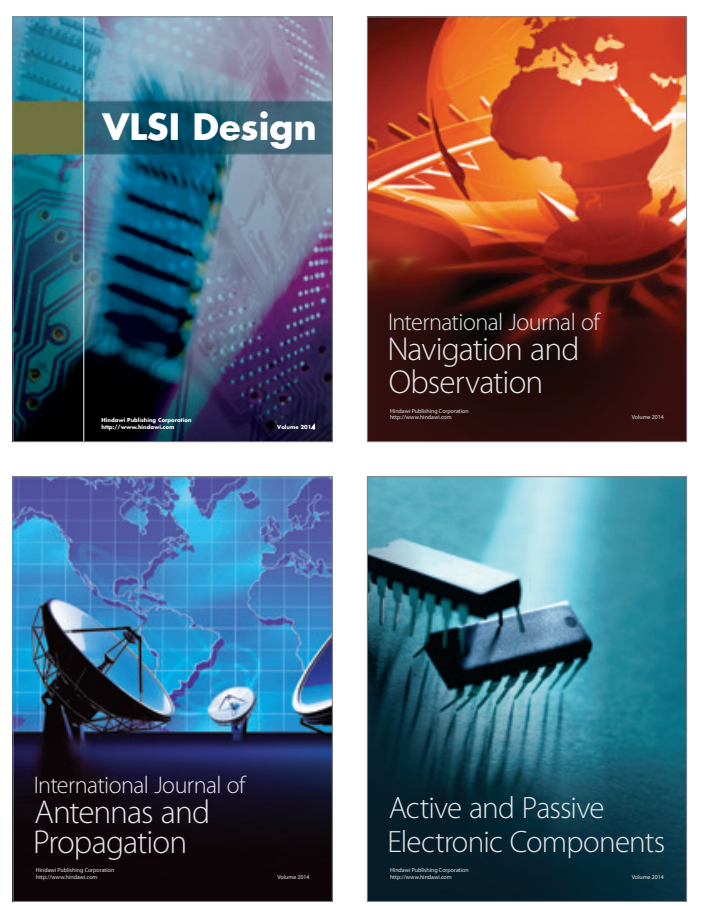
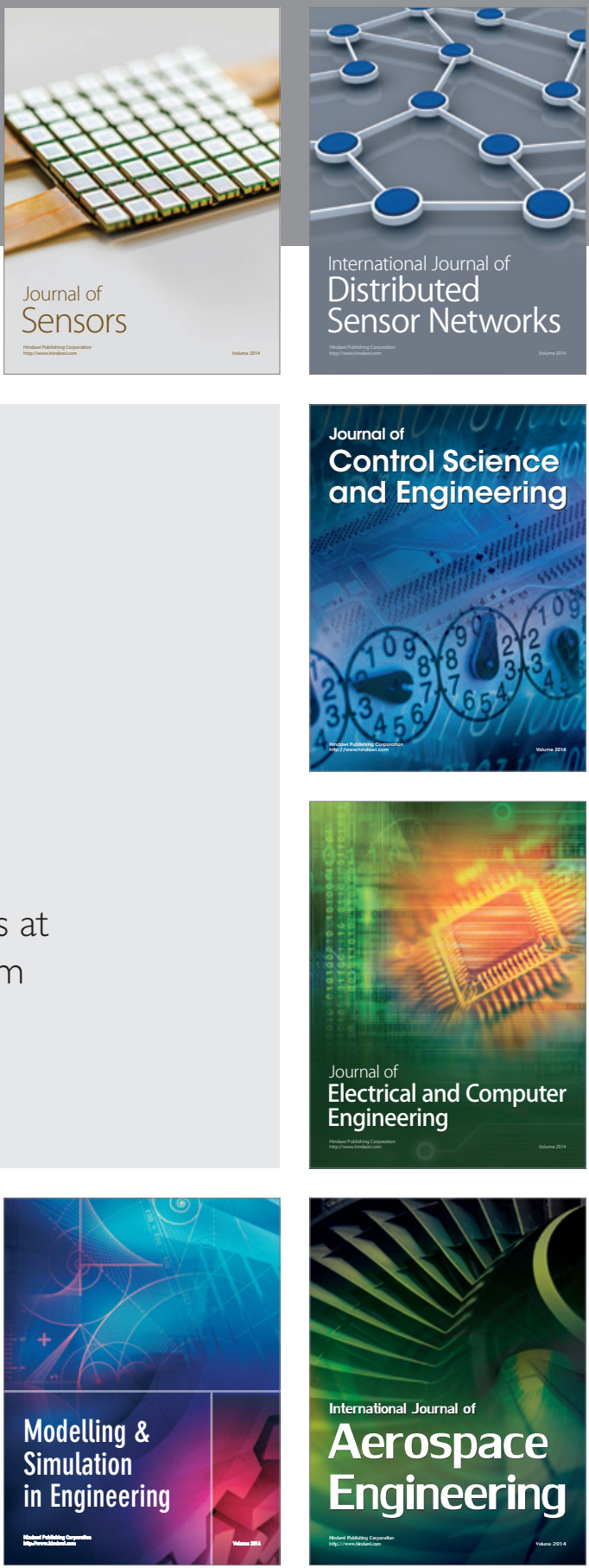

Journal of

Control Science

and Engineering
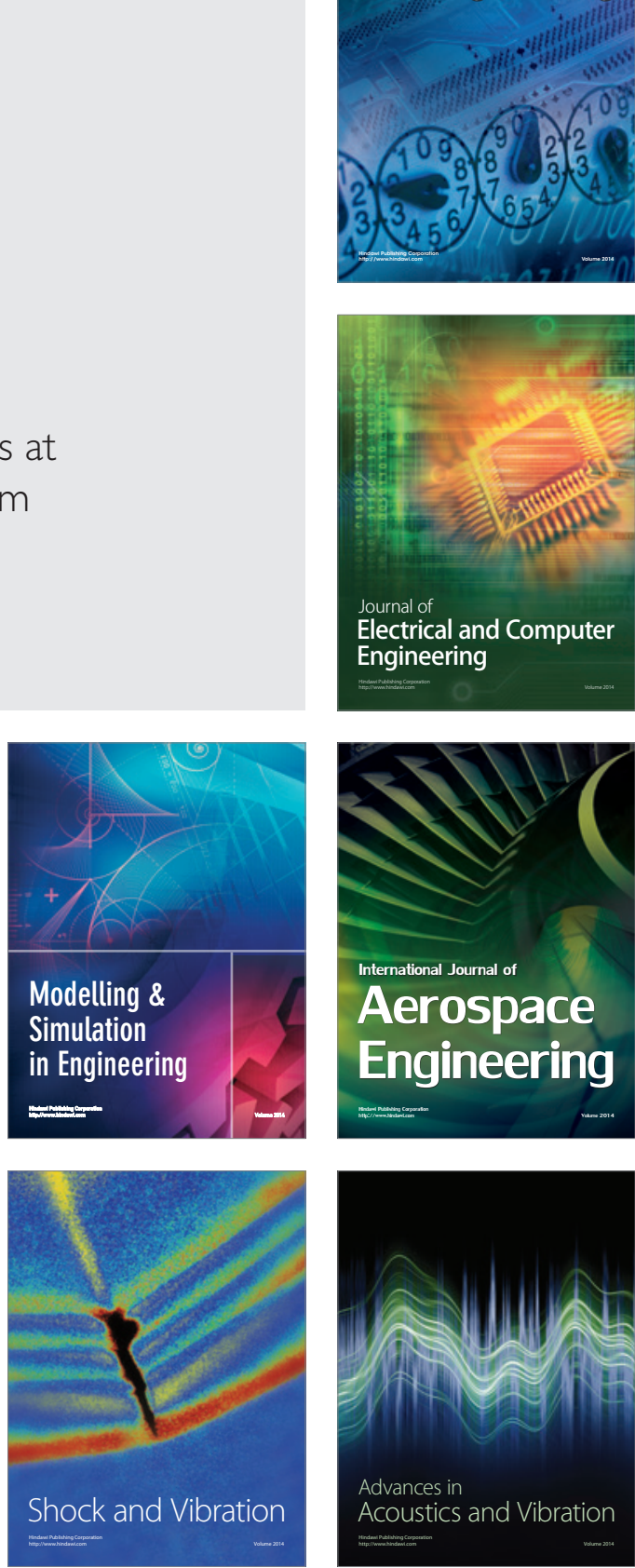